\title{
Preparation and X-Ray Structures of Complexes of 18-Membered Crown Ethers with Polyfunctional Guests: Urea and (O-Alkyliso)Uronium Salts
}

\author{
JOS W. H. M. UITERWIJK ${ }^{\star}$, GERRIT J. VAN HUMMEL ${ }^{a}$, SYBOLT HARKEMA ${ }^{a}$, \\ VERONIKA M. L. J. AARTS $S^{b}$, KARI DAASVATN ${ }^{b}$, JAN GEEVERS ${ }^{b}$, HERMAN J. \\ DEN HERTOG, $\mathbf{J r}^{\mathrm{b}}{ }^{\mathrm{b}}$, and DAVID N. REINHOUDT ${ }^{\mathrm{b}}$ \\ Laboratories of ${ }^{a}$ Chemical Physics and ${ }^{b}$ Organic Chemistry, University of Twente, P.O. Box 217. 7500 AE \\ Enschede, The Netherlands
}

(Received: 1 June 1987; in final form: 25 June 1987)

\begin{abstract}
The preparation and X-ray structure determinations of six complexes of urea and (O-n-butyliso) uronium salts with crown ethers are presented. Urea forms isostructural $5: 1$ adducts with 18-crown-6 (1) and aza-18-crown-6 (2), in which two urea molecules are each hydrogen bonded to two neighbouring hetero atoms of the macroring. The remaining urea molecules form two-dimensional layers alternating with crown ether layers. In both complexes the macroring has the $g^{+} g^{+} a a g^{-} a a g^{-} a g^{-} g^{-} a a g^{+} a a g^{+} a$ conformation with $C_{\mathrm{i}}$ symmetry. In the solid $1: 1$ complex of 0 - $n$-butylisouronium picrate with 18 -crown- 6 (3) two types of conformations of the macroring were observed: the $g^{+} g^{+} a a g^{-} a a g^{+} a a g^{-} g^{-} a g^{-} a a g^{+} a$ conformation with approximate $C_{\mathrm{m}}$ symmetry and to a lesser extent the $g^{+} g^{+} a \mathrm{ag}^{-} a \mathrm{ag}^{+} a g^{+} g^{+} a a g^{-} a \mathrm{ag}^{+} a$ conformation with approximate $C_{2}$ symmetry. Both conformations allow the guest to form three hydrogen bonds to the macrocyclic host. Three complexes of 18 -crown- 6 and uronium salts have been prepared and characterized by $\mathrm{X}$-ray crystallography. The $1: 1$ complexes with uronium nitrate (4) and uronium picrate (5) both exhibit the same $C_{2}$ conformation and the same hydrogen bonding scheme as in the least occupied form of the previous complex. A 1:2 complex with uronium p-toluenesulphonate (6) has a different hydrogen bonding scheme (two hydrogen bonds per cation to neighbouring oxygen atoms of the macroring) and a different conformation of the host molecule (the $a g^{+} a a g^{-} a a^{+} a a g^{-} a g^{+} a g^{-} a$ conformation with almost $D_{3 d}$ symmetry). An attempt to prepare a solid uronium nitrate compiex with diaza-18-crown-6 in the same way as the 18 -crown-6 uronium nitrate $(1: 1)$ complex did not yield the expected result. Instead $\mathrm{X}$-ray analysis revealed that the uronium ion is dissociated, resulting in the nitrate salt of the diprotonated diaza crown ether (7).
\end{abstract}

Key words: Crown ethers, urea(-like) guests, complexation, crystal structures.

Supplementary Data relating to this article are deposited with the British Library as Supplementary Publication No. SUP 82058 (26 pages).

\section{Introduction}

Synthetic receptor molecules for urea are of practical importance for the selective removal of urea in dialysis and could mimic the enzyme urease [1]. We are involved in a study to use macrocyclic polyethers for this purpose, because during the past decade it has been shown that such compounds are capable of forming complexes with organic salts [2] and with neutral organic molecules [3].

\footnotetext{
* Author for correspondence.
} 
In one of the first papers on macrocyclic polyethers Pedersen [4] already reported that urea increases the solubility of dibenzo-18-crown-6 in methanol. He suggested that "there is some interaction", but crystalline complexes of urea with the polyethers could not be obtained. More recently, Vögtle et al. [3f] have reported a crystalline complex of 18 -crown-6 and urea (ratio $1: 4$ ), however without details about the structure of such a complex [5].

In the complexation of urea, two aspects are of particular interest. Firstly, urea would be a neutral guest molecule in the complex and although a number of complexes of 18 -membered crown ethers and neutral species have recently been reported [6], the factors that determine their thermodynamic stabilities are virtually unknown [7]. Secondly, the complexation of urea would be an example of a guest molecule that has different functional groups in close proximity, that might interact with the 18-crown-6 macroring.

Previously we have reported complexation studies of benzo [8] and pyrido [9] crown ethers with the guanidinium cation, which we used as a model compound for the uronium cation (the two cations are isoelectronic). It was shown that crown ethers with 27 or more ring atoms are suited to form encapsulated complexes with the guanidinium cation.

In this paper we report the results of our work on the complexation of urea by 18-membered crown ethers. Furthermore, we have investigated the influence on the complexation of locating a positive charge on the urea molecule, either irreversibly by O-alkylation or by protonation (which is known to occur at the oxygen atom [10]), which in solution represents a reversible process. Similar investigations on the complexation of larger crown ethers with urea [11] and uronium salts [12] have been published separately.

\section{Experimental Section}

The compounds were characterized by their ${ }^{1} \mathrm{H}$ and ${ }^{13} \mathrm{C}$ NMR, mass and infrared spectra as well as elemental analyses. Melting points were recorded on a Reichert mp microscope, the ${ }^{1} \mathrm{H}$ NMR spectra on a Bruker $80 \mathrm{FT}$ and a Varian XL-100 spectrometer in $\mathrm{CDCl}_{3}$ with TMS as internal standard. Mass spectra were obtained with a Varian MAT $311 \mathrm{~A}$ mass spectrometer and IR spectra with a Perkin Elmer 257 spectrometer. Elemental analyses were carried out by the Elemental Analysis Section of the Institute for Organic Chemistry, TNO, Utrecht, The Netherlands, under the supervision of W. J. Buis.

\subsection{STARTING MATERIALS}

Urea (Merck) was recrystallized from $\mathrm{MeOH}$. O-n-butylisouronium picrate [13], uronium nitrate [14] and uronium $p$-toluenesulphonate [15] were prepared according to literature procedures. Uronium picrate was prepared by addition of $0.1 \mathrm{~mol}$ picric acid to $0.1 \mathrm{~mol}$ urea dissolved in $2000 \mathrm{~mL}$ water and heated until a clear solution was obtained. After cooling the salt precipitated in fine crystalline needles. $\mathrm{Mp}$ $\sim 150^{\circ} \mathrm{C}$.

18-Crown-6 was prepared according to Dale et al. [16] and purified by distillation. Aza-18-crown-6 was prepared according to Maeda et al. [17]. Diaza-18-crown-6 (Merck) was used without further purification. 


\subsection{COMPLEXES}

18-Crown-6: urea (1:5) complex (1). $366.6 \mathrm{mg}(1.39 \mathrm{mmol}) 18$-crown-6 and 120.1 $\mathrm{mg}(2.00 \mathrm{mmol})$ urea were dissolved in a mixture of $2 \mathrm{~mL}$ methanol and $2 \mathrm{~mL}$ chloroform. The mixture was heated and after all crystals were dissolved, diethylether was added until the mixture was slightly turbid. Upon cooling colourless crystals were formed $(177.7 \mathrm{mg})$. The crystals were recrystallized from methanol/ethyl acetate giving $85.2 \mathrm{mg}$ of the complex (37.7\%). Mp 145-147 ${ }^{\circ} \mathrm{C}$. Anal. Calcd. for $\mathrm{C}_{17} \mathrm{H}_{44} \mathrm{O}_{11} \mathrm{~N}_{10}: \mathrm{C}, 37.17 ; \mathrm{H}, 7.86 ; \mathrm{N}, 24.81$. Found: C, 36.12; H, 7.88; N, 24.90.

Aza-18-crown-6: urea (1:5) complex (2). $200.0 \mathrm{mg}(0.76 \mathrm{mmol})$ aza-18-crown-6 and $44.9 \mathrm{mg}(0.75 \mathrm{mmol})$ urea were dissolved in a hot mixture of $1 \mathrm{~mL}$ methanol and $2 \mathrm{~mL}$ chloroform. After addition of petroleum ether $(60-80)$ until the mixture became slightly turbid, white crystals precipitated upon cooling. Mp $127-130{ }^{\circ} \mathrm{C}$. Anal. Calcd. for $\mathrm{C}_{17} \mathrm{H}_{45} \mathrm{O}_{10} \mathrm{~N}_{11}$ : C, 36.23; H, 8.05; N, 27.34. Found: C, 36.33; H, $8.22 ; \mathrm{N}, 27.07$.

18-Crown-6: O-n-butylisouronium picrate $(I: 1)$ complex (3). $362.7 \mathrm{mg}$ (1.05 mmol) O- $n$-butylisouronium picrate and $419.7 \mathrm{mg}(1.59 \mathrm{mmol}) 18$-crown-6 were dissolved in $0.5 \mathrm{~mL}$ hot methanol and $1 \mathrm{~mL}$ of chloroform. By careful addition of $3 \mathrm{~mL}$ diethylether, $382.3 \mathrm{mg}$ of a yellow precipitate was formed which after recrystallization from ethyl acetate gave $342.4 \mathrm{mg}$ of the $1: 1$ complex $(53.5 \%)$. Mp $95-97^{\circ} \mathrm{C}$. Anal. Calcd. for $\mathrm{C}_{23} \mathrm{H}_{39} \mathrm{O}_{14} \mathrm{~N}_{5}$ : C, 45.32; H, 6.40; N, 11.49. Found: C, 45.30; $\mathrm{H}$, $6.49 ; \mathrm{N}, 11.48$.

18-Crown-6: uronium nitrate $(1: 1)$ complex (4). To $147.7 \mathrm{mg}(1.20 \mathrm{mmol})$ uronium nitrate and $414.7 \mathrm{mg}(1.57 \mathrm{mmol}) 18$-crown-6, dissolved in $0.5 \mathrm{~mL}$ hot methanol, $1 \mathrm{~mL} \mathrm{CHCl}_{3}$ was added followed by $3 \mathrm{~mL}$ of diethyl ether. $202.8 \mathrm{mg}$ of a crystalline compound was formed which upon recrystallization from methanol/ethyl acetate gave $119.2 \mathrm{mg}$ of the $1: 1$ complex $(25.6 \%)$. Mp 88-91 ${ }^{\circ} \mathrm{C}$. Anal. Calcd. for $\mathrm{C}_{13} \mathrm{H}_{29^{-}}$ $\mathrm{O}_{10} \mathrm{~N}_{3}: \mathrm{C}, 40.31 ; \mathrm{H}, 7.55 ; \mathrm{N}, 10.85$. Found: $\mathrm{C}, 40.42 ; \mathrm{H}, 7.52 ; \mathrm{N}, 10.64$.

18-Crown-6: uronium picrate $(1: 1)$ complex (5). To a solution of $426.7 \mathrm{mg}$ (1.61 mmol) 18 -crown-6 and $291.0 \mathrm{mg}(1.01 \mathrm{mmol})$ uronium picrate in $0.4 \mathrm{~mL}$ methanol and $0.8 \mathrm{~mL}$ chloroform $2 \mathrm{~mL}$ diethyl ether was added, giving a yellow crystalline precipitate $(309.1 \mathrm{mg})$. The complex was purified by recrystallization from hot methanol/ethyl acetate. $286.0 \mathrm{mg}$ of a yellow crystalline $(1: 1)$ complex was isolated (51.2\%). Mp $128-130{ }^{\circ} \mathrm{C}$. Anal. Calcd. for $\mathrm{C}_{19} \mathrm{H}_{31} \mathrm{O}_{14} \mathrm{~N}_{5}: \mathrm{C}, 41.23 ; \mathrm{H}, 5.65 ; \mathrm{N}$, 12.65. Found: C, $40.45 ; \mathrm{H}, 5.48 ; \mathrm{N}, 13.47$.

18-Crown-6: uronium p-toluenesulphonate (1:2) complex (6). To $230.0 \mathrm{mg}(0.99$ $\mathrm{mmol})$ of uronium $p$-toluenesulphonate and $403.9 \mathrm{mg}(1.53 \mathrm{mmol}) 18$-crown-6 dissolved in $2 \mathrm{~mL}$ hot methanol, $4 \mathrm{~mL} \mathrm{CHCl}_{3}$ was added followed by $5 \mathrm{~mL}$ diethyl ether. A white crystalline precipitate was formed which was carefully washed with $\mathrm{CCl}_{4}$ and after drying gave $282.3 \mathrm{mg}$ of colourless crystals $(78.2 \%)$. Mp $117-119{ }^{\circ} \mathrm{C}$. Anal. Calcd. for $\mathrm{C}_{28} \mathrm{H}_{48} \mathrm{O}_{14} \mathrm{~N}_{4} \mathrm{~S}_{2}$ : C, 46.15; H, 6.59; N, 7.69; S, 8.80. Found: C, $46.19 ; \mathrm{H}, 6.62 ; \mathrm{N}, 7.71 ; \mathrm{S}, 8.60$. 


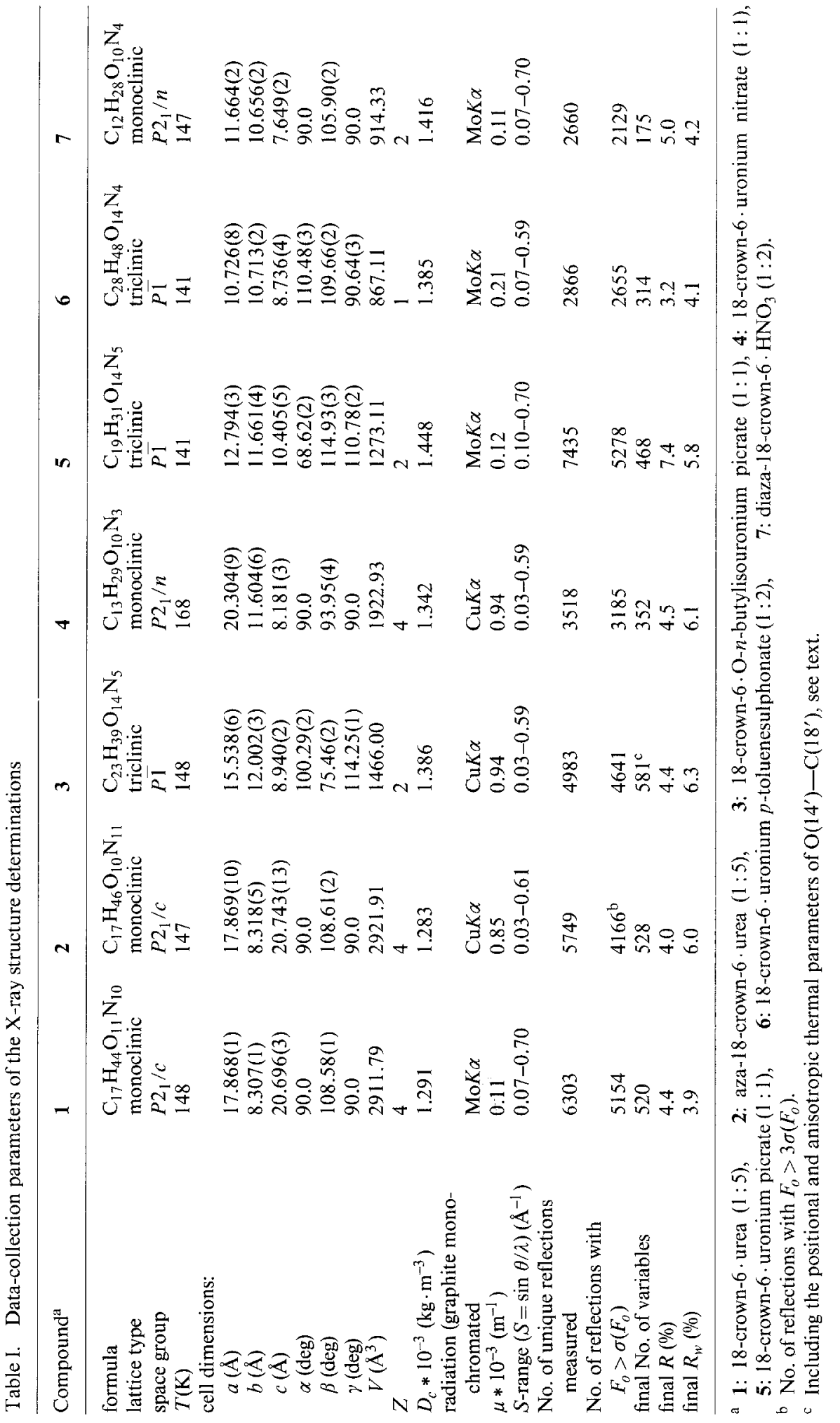


Diaza-18-crown-6: $\mathrm{HNO}_{3}(1: 2)$ (compound 7). $200.0 \mathrm{mg}(0.76 \mathrm{mmol}$ ) of diaza-18crown-6 and $45.0 \mathrm{mg}(0.75 \mathrm{mmol})$ uronium nitrate were dissolved in a hot solution of $1 \mathrm{~mL}$ methanol and $2 \mathrm{~mL}$ chloroform. Diethyl ether was added until the mixture was slightly turbid. $111.5 \mathrm{mg}$ of a white precipitate was formed, which appeared to be the dinitrate salt of diaza-18-crown-6. Mp 179-182 ${ }^{\circ} \mathrm{C}$. Anal. Calcd. for $\mathrm{C}_{12} \mathrm{H}_{28} \mathrm{O}_{10} \mathrm{~N}_{4}$ : C, 37.11; H, 7.15; N, 14.43. Found: C, 36.33; H, 7.12; N, 14.88.

\subsection{X-RAY DIFFRACTION}

X-Ray measurements were performed on a single-crystal diffractometer (Philips PW1100) using the $\omega-2 \theta$ scanning mode. The most important data-collection parameters are presented in Table I. Measured intensities were corrected for the decrease in intensity during data-collection, using the intensities of three standard reflections, measured every hour.

The structures were solved by direct methods [18] and refined by full-matrix leastsquares [19], using reflections having an intensity greater than the estimated standard deviation from counting statistics (and three times this deviation for complex 2). For all complexes, except 3 , all hydrogens could unambiguously be located irom difference-Fourier maps. In the complex of aza-18-crown-6 with urea (2) the ring nitrogen atoms are disordered. Difference-Fourier syntheses revealed all hydrogens attached to the nitrogen atoms of urea and the carbon atoms of the ring. After inclusion of these hydrogen atoms in the refinement a new difference-Fourier synthesis showed two 'half hydrogens' in each independent ring, fixing the positions of the nitrogen atoms in the ring. For the complex of 18 -crown- 6 with $\mathrm{O}$ - $n$-butylisouronium picrate (3) part of the macroring was found to be disordered in an initial state of refinement. It was possible to describe this disorder by attributing two different positions for five ring atoms $\left(\mathrm{O}(14)-\mathrm{C}(18)\right.$ and $\mathrm{O}\left(14^{\prime}\right)-\mathrm{C}\left(18^{\prime}\right)$, see Table II), with occupancies of $72.1 \%$ and $27.9 \%$. In this part hydrogens could only be located for the highest occupied state.

Parameters refined were the overall scaling factor, isotropic extinction parameter, positional parameters of all atoms, anisotropic thermal parameters for heavy atoms (including the lowest occupied ring atoms $\mathrm{O}\left(14^{\prime}\right)-\mathrm{C}\left(18^{\prime}\right)$ in complex 3) and isotropic thermal parameters for hydrogens. The weight for each reflection was taken to be $w=\left\{\sigma\left(F_{0}\right)+0.01\left|F_{0}\right|\right\}^{-2}$, where $\sigma\left(F_{0}\right)$ is the estimated standard deviation of the observed structure factor $\left(F_{0}\right)$ derived from counting statistics. Scattering factors for heavy atoms were taken from International Tables for X-ray Crystallography [20]; for $\mathrm{H}$ the scattering factor tables of Stewart, Davidson and Simpson [21] were used. No absorption corrections were applied.

\section{Results}

\subsection{COMPLEXATION OF UREA}

Unlike similar experiments with alkylammonium salts [2a, 22], the equilibration of aqueous solutions of urea with solutions of crown ethers $(n \leqslant 18)$ in chloroform did not result in a measurable transfer of urea from the aqueous to the organic phase. However, when to a solution of 18-crown-6 and urea in hot methanol/chloroform diethylether was added after cooling, colourless crystals precipitated that were re- 
crystallized from methanol/ethyl acetate. The material had a well-defined melting range of $146-148{ }^{\circ} \mathrm{C}$ and analyzed correctly for an adduct of urea and 18-crown-6 in a ratio of $5: 1$ (complex 1). In a similar way a $5: 1$ complex of urea and aza-18crown -6 could be formed, which precipitated upon addition of petroleum ether $(60-80)$. The white crystals had a melting range of $127-130{ }^{\circ} \mathrm{C}$ ( complex 2). (Di)benzo-18-crown-6, dicyclohexano-18-crown-6 and crown ethers with less than 18 ring atoms, on the contrary, gave no solid adducts with urea using similar procedures.

Determinations of the thermodynamic stability constants of $1: 1$ complexes of urea and 18-crown-6 in methanol by polarography has not provided accurate data, but preliminary results indicate $\log K_{a}$ values smaller than one [23].

\subsection{COMPLEXATION OF O-n-BUTYL-ISOURONIUM PICRATE}

In view of the low thermodynamic stability of the neutral urea 18 -crown-6 $(1: 5)$ complex we have investigated the effect of alkylation of urea. Previously, we have described extraction experiments and dynamic ${ }^{1} \mathrm{H}$ NMR studies of various crown ether.S-tert-butyl-isothiouronium salt complexes [24]. Adducts with different host/ guest ratios could be obtained in the solid state. The X-ray structures of the perchlorate salt complexes with 18-crown-6 [25] (2:1 ratio) and with 1,3-xylyl-18crown-5 [24] (1:1 ratio) have been determined, revealing perching complexes, in which the guests are hydrogen-bonded via two or three hydrogen bonds to the host, with additional hydrogen bonds to the anions. Similar complexes can be expected with alkylated urea salts.

The complex of $\mathrm{O}$-n-butyl-isouronium picrate with 18-crown-6 was prepared from a hot solution of 18-crown-6 and the salt in methanol. The complex was obtained in $54 \%$ yield. ${ }^{1} \mathrm{H}$ NMR Spectroscopy and elemental analysis proved that the host/guest ratio was $1: 1$ (complex 3 ).

\subsection{COMPLEXATION OF URONIUM SALTS}

The complexation studies described above with $\mathrm{O}$ - (or S)-alkyliso(thio) uronium salts have the advantage that the guest molecule in the complex can be studied by ${ }^{1} \mathrm{H}$ NMR spectroscopy. The disadvantage, however, is the increased size of the guest molecule, which may be a hindrance in complexation. Another method considered to enhance the stability of urea complexes is protonation of the guest, a method which lacks the disadvantage of the former method. Protonation converts the urea in a polyfunctional cation, isoelectronic with guanidinium, which is known to form welldefined stoichiometric complexes with 18-crown-6 [26]. Therefore three complexes of 18 -crown- 6 with uronium salts have been prepared and the interactions between host and guest were determined by X-ray crystallography.

The complexes were prepared from hot methanolic solutions and analyzed correctly for 1:1 molecular complexes in the cases of uronium nitrate (complex 4) and picrate (complex 5), and a 1:2 complex for the uronium $p$-toluenesulphonate salt (complex 6).

When a mixture of diaza-18-crown-6 and uronium nitrate, dissolved in hot methanol/chloroform, was treated after cooling with diethylether, a white solid precipitated, which analyzed correctly for the dinitrate salt of diaza-18-crown-6 (compound 7). This result demonstrates that protonation of the polyether nitrogen 
atoms (with dissociation of the uronium cation) without complexation of the urea formed is preferred over complexation of the uronium cation as observed in the analogous experiments involving 18 -crown-6.

\subsection{X-RAY ANALYSIS}

The crystal and molecular structures of the compounds 1-7 have been determined by X-ray diffraction [27]. Stereoscopic ORTEP [28] views of the compounds, including atom numberings, are presented in Figures 1-7, using 50\% probability ellipsoids for all non-hydrogen atoms. The radii of the hydrogen atoms have been chosen arbitrarily. Positional and equivalent isotropic thermal parameters [29] of the non-hydrogen atoms are collected in Table II. Torsion angles in the macrorings are given in Table III. H-Bond parameters are presented in Table IV.

Table II. Fractional co-ordinates ${ }^{\mathrm{a}}\left(\times 10^{4}\right)$ and equivalent isotropic thermal parameters ${ }^{\mathrm{a}, \mathrm{b}}\left(\times 10^{4}\right)$ of the non-hydrogen atoms in the compounds ${ }^{\mathrm{c}}$

\begin{tabular}{|c|c|c|c|c|}
\hline \multicolumn{5}{|c|}{1} \\
\hline Atom & $x / a$ & $y / b$ & $z / c$ & $U_{\mathrm{cq}}$ \\
\hline$C(1)$ & $-605(1)$ & 1411212 & $707(1)$ & $31(1)$ \\
\hline $0(2)$ & $-1107(1)$ & $12805(1)$ & $383(1)$ & $27(1)$ \\
\hline$C(3)$ & $-1179(1)$ & $11548(2)$ & $829(1)$ & $30(1)$ \\
\hline$C(4)$ & $-1683(1)$ & $10248(2)$ & $406(1)$ & $30(1)$ \\
\hline $0(5)$ & $-1267(1)$ & $9547(1)$ & $-6(1)$ & $27(1)$ \\
\hline$C(6)$ & $-1706(1)$ & $8291(2)$ & $-422(1)$ & $31(1)$ \\
\hline$C(7)$ & $-1315(1)$ & $7762(2)$ & $-925(1)$ & $30(1)$ \\
\hline$O(8)$ & $-564(1)$ & $7059(1)$ & $-584(1)$ & $27(1)$ \\
\hline$C(9)$ & $-236(1)$ & $6363(2)$ & $-1060(1)$ & $30(1)$ \\
\hline$C(10)$ & $5668(1)$ & $7717(2)$ & $4082(1)$ & $41(1)$ \\
\hline $0(11)$ & $5150(1)$ & $7097(1)$ & $4418(1)$ & $35(1)$ \\
\hline$C(12)$ & $4451(1)$ & $6467(2)$ & $3941(1)$ & $45(1)$ \\
\hline$C(1.3)$ & $3865(1)$ & $6066(2)$ & $4296(1)$ & $45(1)$ \\
\hline $0(14)$ & $3599(1)$ & $7424(1)$ & $4590(1)$ & $38(1)$ \\
\hline$C(15)$ & $3212(1)$ & $8650(2)$ & $4124(1)$ & $43(1)$ \\
\hline$C(16)$ & $2990(1)$ & $9952(2)$ & $4522(1)$ & $41(1)$ \\
\hline$o(17)$ & $3698(1)$ & $10586(1)$ & $4980(1)$ & $30(1)$ \\
\hline$c(18)$ & $3568(1)$ & $11819(2)$ & $5404(1)$ & $41(1)$ \\
\hline$C(19)$ & $1087(1)$ & $7853(2)$ & $1380(1)$ & $20(1)$ \\
\hline$O(20)$ & $1299(1)$ & $7976(1)$ & $2021(1)$ & $21(1)$ \\
\hline$N(21)$ & $1601(1)$ & $7397(2)$ & $1066(1)$ & $28(1)$ \\
\hline$N(22)$ & $336(1)$ & $8139(2)$ & $999(1)$ & $28(1)$ \\
\hline$C(23)$ & $5056(1)$ & $7692(2)$ & $6373(1)$ & $22(1)$ \\
\hline$O(24)$ & $5325(1)$ & $7432(1)$ & $7005(1)$ & $23(1)$ \\
\hline$N(25)$ & $5547(1)$ & $7917(2)$ & $6009(1)$ & $32(1)$ \\
\hline$N(26)$ & $4283(1)$ & $7757(2)$ & $6051(1)$ & $33(1)$ \\
\hline$c(27)$ & $3421(1)$ & $6114(2)$ & $7196(1)$ & $21(1)$ \\
\hline $0(28)$ & $3254(1)$ & $7458(1)$ & $6901(1)$ & $23(1)$ \\
\hline N $(29)$ & $2858(1)$ & $5254(2)$ & $7345(1)$ & $30(1)$ \\
\hline$N(30)$ & $4149(1)$ & $5504(2)$ & $7366(1)$ & $28(1)$ \\
\hline$C(31)$ & $-2681(1)$ & $8934(2)$ & $2207(1)$ & $21(1)$ \\
\hline $0(32)$ & $-2765(1)$ & $7628(1)$ & $1883(1)$ & $23(1)$ \\
\hline$N(33)$ & $-2046(1)$ & $9873(2)$ & $2270(1)$ & $27(1)$ \\
\hline$N(34)$ & $-3213(1)$ & $9423(2)$ & $2495(1)$ & $28(1)$ \\
\hline$C(35)$ & $-636(1)$ & $6865(2)$ & $2161(1)$ & $18(1)$ \\
\hline
\end{tabular}


Table II-Continued

\begin{tabular}{lrlll}
\hline$O(36)$ & $-740(1)$ & $8233(1)$ & $1888(1)$ & $22(1)$ \\
$N(37)$ & $90(1)$ & $6235(2)$ & $2422(1)$ & $23(1)$ \\
$N(38)$ & $-1249(1)$ & $5973(2)$ & $2299(1)$ & $25(1)$
\end{tabular}

2

\begin{tabular}{|c|c|c|c|c|}
\hline Atom & $x / a$ & $y / b$ & $z / c$ & $U_{\mathrm{eq}}$ \\
\hline$C(1)$ & $-628(1)$ & $14096(3)$ & $702(1)$ & $38(1)$ \\
\hline$O(2)$ & $-1131(1)$ & $12794(2)$ & $381(1)$ & $34(1)$ \\
\hline$C(3)$ & $-1209(1)$ & $11545(3)$ & $323(1)$ & $39(1)$ \\
\hline$C(4)$ & $-1702(1)$ & $10244(3)$ & $403(1)$ & $37(1)$ \\
\hline$O(5)$ & $-1285(1)$ & $9576(2)$ & $-12(1)$ & $36(1)$ \\
\hline$C(6)$ & $-1713(1)$ & $8307(3)$ & $-425(1)$ & $39(1)$ \\
\hline$c(7)$ & $-1317(1)$ & $7787(3)$ & $-924(1)$ & $39(1)$ \\
\hline ON ( 8) & $-535(1)$ & $7178(2)$ & $-584(1)$ & $38(1)$ \\
\hline$C(9)$ & $-218(1)$ & $6392(3)$ & $-1054(1)$ & $37(1)$ \\
\hline$C(10)$ & $5664(2)$ & $7753(3)$ & $4077(1)$ & $53(1)$ \\
\hline ON (11) & $5108(1)$ & $7228(3)$ & $4403(1)$ & $53(1)$ \\
\hline$C(12)$ & $4421(2)$ & $6512(3)$ & $3928(1)$ & $60(1)$ \\
\hline$c(13)$ & $3841(2)$ & $6086(3)$ & $4278(1)$ & $60(1)$ \\
\hline $0(14)$ & $3570(1)$ & $7439(2)$ & $4576(1)$ & $51(1)$ \\
\hline$c(15)$ & $3188(2)$ & $8680(4)$ & $4120(1)$ & $58(1)$ \\
\hline$c(16)$ & $2977(1)$ & $9963(4)$ & $4522(1)$ & $56(1)$ \\
\hline$O(17)$ & $3693(1)$ & $10560(2)$ & $4992(1)$ & $43(1)$ \\
\hline$C(18)$ & $3569(1)$ & $11794(3)$ & $5410(1)$ & $54(1)$ \\
\hline$C(19)$ & $1078(1)$ & $7821(2)$ & $1388(1)$ & $25(1)$ \\
\hline $0(20)$ & $1304(1)$ & $7964(2)$ & $2029(1)$ & $26(1)$ \\
\hline$N(21)$ & $1589(1)$ & $7361(2)$ & $1070(1)$ & $33(1)$ \\
\hline$N(22)$ & $329(1)$ & $8103(2)$ & $1016(1)$ & $35(1)$ \\
\hline$C(23)$ & $5061(1)$ & $7656(2)$ & $6371(1)$ & $28(1)$ \\
\hline $0(24)$ & $5324(1)$ & $7455(2)$ & $7006(1)$ & $29(1)$ \\
\hline$N(25)$ & $5557(1)$ & $7870(3)$ & $6011(1)$ & $42(1)$ \\
\hline$N(26)$ & $4286(1)$ & $7670(3)$ & $6039(1)$ & $39(1)$ \\
\hline$C(27)$ & $3418(1)$ & $6121(2)$ & $7191(1)$ & $25(1)$ \\
\hline$O(28)$ & $3253(1)$ & $7462(2)$ & $6894(1)$ & $29(1)$ \\
\hline$N(29)$ & $2862(1)$ & $5275(2)$ & $7342(1)$ & $35(1)$ \\
\hline$N(30)$ & $4148(1)$ & $5518(2)$ & $7360(1)$ & $35(1)$ \\
\hline$C(31)$ & $-2679(1)$ & $8920(2)$ & $2219(1)$ & $26(1)$ \\
\hline$O(32)$ & $-2765(1)$ & $7614(2)$ & $1899(1)$ & $29(1)$ \\
\hline$N(33)$ & $-2042(1)$ & $9856(2)$ & $2286(1)$ & $33(1)$ \\
\hline$N(34)$ & $-3216(1)$ & $9416(2)$ & $2501(1)$ & $32(1)$ \\
\hline$c(35)$ & $-636(1)$ & $6840(2)$ & $2165(1)$ & $23(1)$ \\
\hline $0(36)$ & $-739(1)$ & $8209(2)$ & $1896(1)$ & $27(1)$ \\
\hline$N(37)$ & $87(1)$ & $6208(2)$ & $2422(1)$ & $28(1)$ \\
\hline$N(38)$ & $-1252(1)$ & $5952(2)$ & $2201(1)$ & $29(1)$ \\
\hline
\end{tabular}

3

\begin{tabular}{llrrr}
\hline Atom & $x / a$ & $y / b$ & $z / c$ & $U_{\text {eq }}$ \\
\hline$C(1)$ & $4876(1)$ & $9377(2)$ & $3148(2)$ & $34(1)$ \\
$O(2)$ & $4519(1)$ & $8428(1)$ & $1994(1)$ & $29(1)$ \\
$C(3)$ & $3898(1)$ & $7305(2)$ & $2652(2)$ & $31(1)$ \\
$C(4)$ & $3478(1)$ & $6417(2)$ & $1444(2)$ & $28(1)$ \\
$O(5)$ & $4091(1)$ & $6087(1)$ & $269(1)$ & $26(1)$ \\
$C(6)$ & $3687(1)$ & $5406(2)$ & $-1025(2)$ & $28(1)$ \\
$C(7)$ & $4436(1)$ & $5039(2)$ & $-2173(2)$ & $31(1)$
\end{tabular}


Table II - Continued

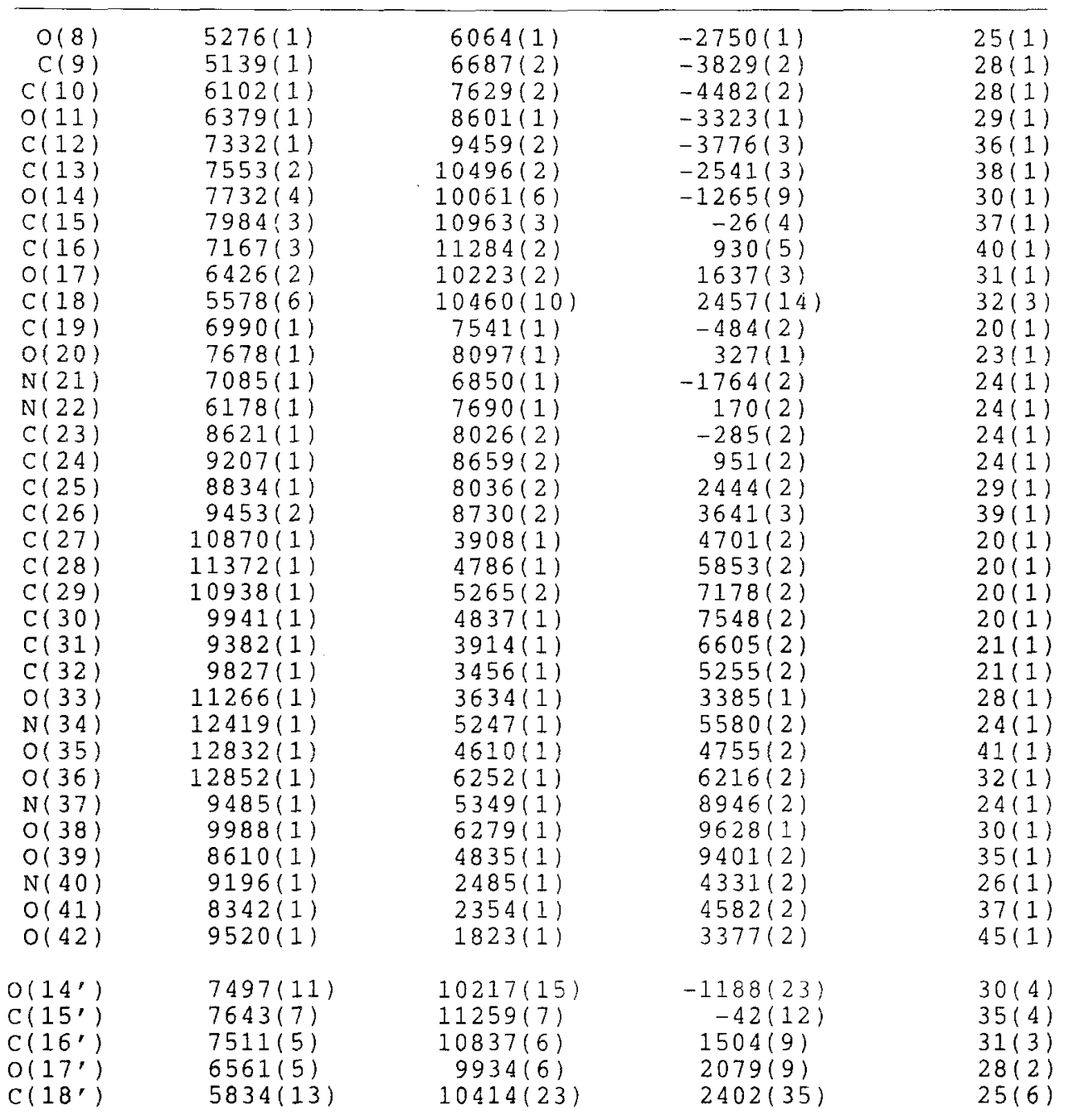

4

\begin{tabular}{llrll}
\hline Atom & $x / a$ & $y / b$ & $z / c$ & $U_{\text {eq }}$ \\
\hline$C(1)$ & $5416(1)$ & $-3285(2)$ & $4813(3)$ & $45(1)$ \\
$O(2)$ & $5007(1)$ & $-3183(1)$ & $6143(2)$ & $41(1)$ \\
$C(3)$ & $5338(1)$ & $-2695(2)$ & $7588(3)$ & $52(1)$ \\
$C(4)$ & $5361(1)$ & $-1416(2)$ & $7553(3)$ & $48(1)$ \\
$O(5)$ & $4701(1)$ & $-983(1)$ & $7476(2)$ & $39(1)$ \\
$C(6)$ & $4690(1)$ & $239(2)$ & $7546(3)$ & $46(1)$ \\
$C(7)$ & $3997(1)$ & $653(2)$ & $7429(3)$ & $45(1)$ \\
$O(8)$ & $3728(1)$ & $534(1)$ & $5792(2)$ & $33(1)$ \\
$C(9)$ & $3080(1)$ & $1006(2)$ & $5568(3)$ & $47(1)$ \\
$C(10)$ & $2815(1)$ & $789(2)$ & $3858(3)$ & $45(1)$ \\
$O(11)$ & $2638(1)$ & $-394(1)$ & $3709(2)$ & $34(1)$ \\
$C(12)$ & $2390(1)$ & $-722(2)$ & $2093(3)$ & $43(1)$ \\
$C(13)$ & $2927(1)$ & $-972(2)$ & $984(3)$ & $43(1)$
\end{tabular}


Table II - Continued

\begin{tabular}{llrrr}
\hline$O(14)$ & $3294(1)$ & $-1922(1)$ & $1643(2)$ & $37(1)$ \\
$C(15)$ & $3778(1)$ & $-2309(2)$ & $602(3)$ & $46(1)$ \\
$C(16)$ & $4161(1)$ & $-3268(2)$ & $1402(3)$ & $45(1)$ \\
$O(17)$ & $4591(1)$ & $-2848(1)$ & $2708(2)$ & $37(1)$ \\
$C(18)$ & $5021(1)$ & $-3724(2)$ & $3358(3)$ & $47(1)$ \\
$C(19)$ & $1736(1)$ & $1854(1)$ & $-937(2)$ & $26(1)$ \\
$O(20)$ & $2350(1)$ & $1537(1)$ & $-822(2)$ & $38(1)$ \\
$N(21)$ & $1552(1)$ & $2893(1)$ & $-556(2)$ & $35(1)$ \\
$N(22)$ & $1308(1)$ & $1063(1)$ & $-1465(2)$ & $35(1)$ \\
$N(23)$ & $8407(1)$ & $1304(1)$ & $4086(2)$ & $29(1)$ \\
$O(24)$ & $8289(1)$ & $2168(1)$ & $4986(2)$ & $31(1)$ \\
$O(25)$ & $8089(1)$ & $1180(1)$ & $2748(2)$ & $46(1)$ \\
$O(26)$ & $8838(1)$ & $617(1)$ & $4560(2)$ & $49(1)$
\end{tabular}

5

\begin{tabular}{|c|c|c|c|c|}
\hline Atom & $x / a$ & $y / b$ & $z / c$ & $U_{\mathrm{ec}}$ \\
\hline$C(1)$ & $211(3)$ & $-7337(3)$ & $18984(3)$ & $36(1)$ \\
\hline$O(2)$ & $410(1)$ & $-8055(2)$ & $18295(2)$ & $31(1)$ \\
\hline$C(3)$ & $-644(2)$ & $-8615(3)$ & $17279(3)$ & $37(1)$ \\
\hline$C(4)$ & $-1072(2)$ & $-7682(3)$ & $15853(3)$ & $35(1)$ \\
\hline $0(5)$ & $-201(1)$ & $-7214(2)$ & $15160(2)$ & $31(1)$ \\
\hline$c(6)$ & $-592(2)$ & $-6390(3)$ & $13743(3)$ & $33(1)$ \\
\hline$C(7)$ & $274(2)$ & $-6050(3)$ & $12974(3)$ & $30(1)$ \\
\hline $0(8)$ & $1315(1)$ & $-5192(2)$ & $13611(2)$ & $26(1)$ \\
\hline$c(9)$ & $2165(2)$ & $-4817(3)$ & $12901(3)$ & $28(1)$ \\
\hline$C(10)$ & $3276(2)$ & $-4010(2)$ & $13718(3)$ & $27(1)$ \\
\hline$O(11)$ & $3795(1)$ & $-4807(1)$ & $15101(2)$ & $25(1)$ \\
\hline$C(12)$ & $4795(2)$ & $-4136(3)$ & $16060(3)$ & $29(1)$ \\
\hline$C(13)$ & $4460(3)$ & $-3579(2)$ & $16887(3)$ & $31(1)$ \\
\hline $0(14)$ & $4007(1)$ & $-4584(2)$ & $17932(2)$ & $27(1)$ \\
\hline$C(15)$ & $3747(2)$ & $-4125(3)$ & $18826(3)$ & $31(1)$ \\
\hline$C(16)$ & $3166(2)$ & $-5205(3)$ & $19804(3)$ & $34(1)$ \\
\hline$O(17)$ & $1985(1)$ & $-5739(2)$ & $18992(2)$ & $30(1)$ \\
\hline$C(18)$ & $1382(3)$ & $-6718(3)$ & $19909(3)$ & $38(1)$ \\
\hline$C(19)$ & $6909(2)$ & $-2462(2)$ & $12197(3)$ & $22(1)$ \\
\hline$O(20)$ & $5809(1)$ & $-2619(2)$ & $12082(2)$ & $25(1)$ \\
\hline$N(21)$ & $7327(2)$ & $-1702(2)$ & $11144(3)$ & $30(1)$ \\
\hline$N(22)$ & $7576(2)$ & $-3049(2)$ & $13439(2)$ & $26(1)$ \\
\hline$C(23)$ & $4401(2)$ & $-678(2)$ & $8382(2)$ & $22(1)$ \\
\hline$C(24)$ & $4677(2)$ & $82(2)$ & $7060(3)$ & $24(1)$ \\
\hline$C(25)$ & $3894(2)$ & $663(2)$ & $5826(3)$ & $26(1)$ \\
\hline$C(26)$ & $2764(2)$ & $498(2)$ & $5787(2)$ & $24(1)$ \\
\hline$C(27)$ & $2384(2)$ & $-273(2)$ & $6958(3)$ & $25(1)$ \\
\hline$c(28)$ & $3183(2)$ & $-830(2)$ & $8201(2)$ & $23(1)$ \\
\hline$O(29)$ & $5146(2)$ & $-1146(2)$ & $9524(2)$ & $33(1)$ \\
\hline$N(30)$ & $5833(2)$ & $258(2)$ & $6986(2)$ & $35(1)$ \\
\hline $0(31)$ & $6485(2)$ & $-418(3)$ & $7900(3)$ & $90(2)$ \\
\hline $0(32)$ & $6092(2)$ & $1013(2)$ & $5935(2)$ & $39(1)$ \\
\hline$N(33)$ & $1942(2)$ & $1133(2)$ & $4480(2)$ & $33(1)$ \\
\hline $0(34)$ & $2336(2)$ & $1882(2)$ & $3502(2)$ & $48(1)$ \\
\hline $0(35)$ & $915(2)$ & $892(2)$ & $4406(2)$ & $44(1)$ \\
\hline $\mathrm{N}(36)$ & $2740(2)$ & $-1623(2)$ & $9406(2)$ & $33(1)$ \\
\hline$O(37)$ & $1691(2)$ & $-1933(4)$ & $9160(3)$ & $105(2)$ \\
\hline$O(38)$ & $3401(2)$ & $-2002(3)$ & $10580(3)$ & $89(2)$ \\
\hline
\end{tabular}


Table II - Continued

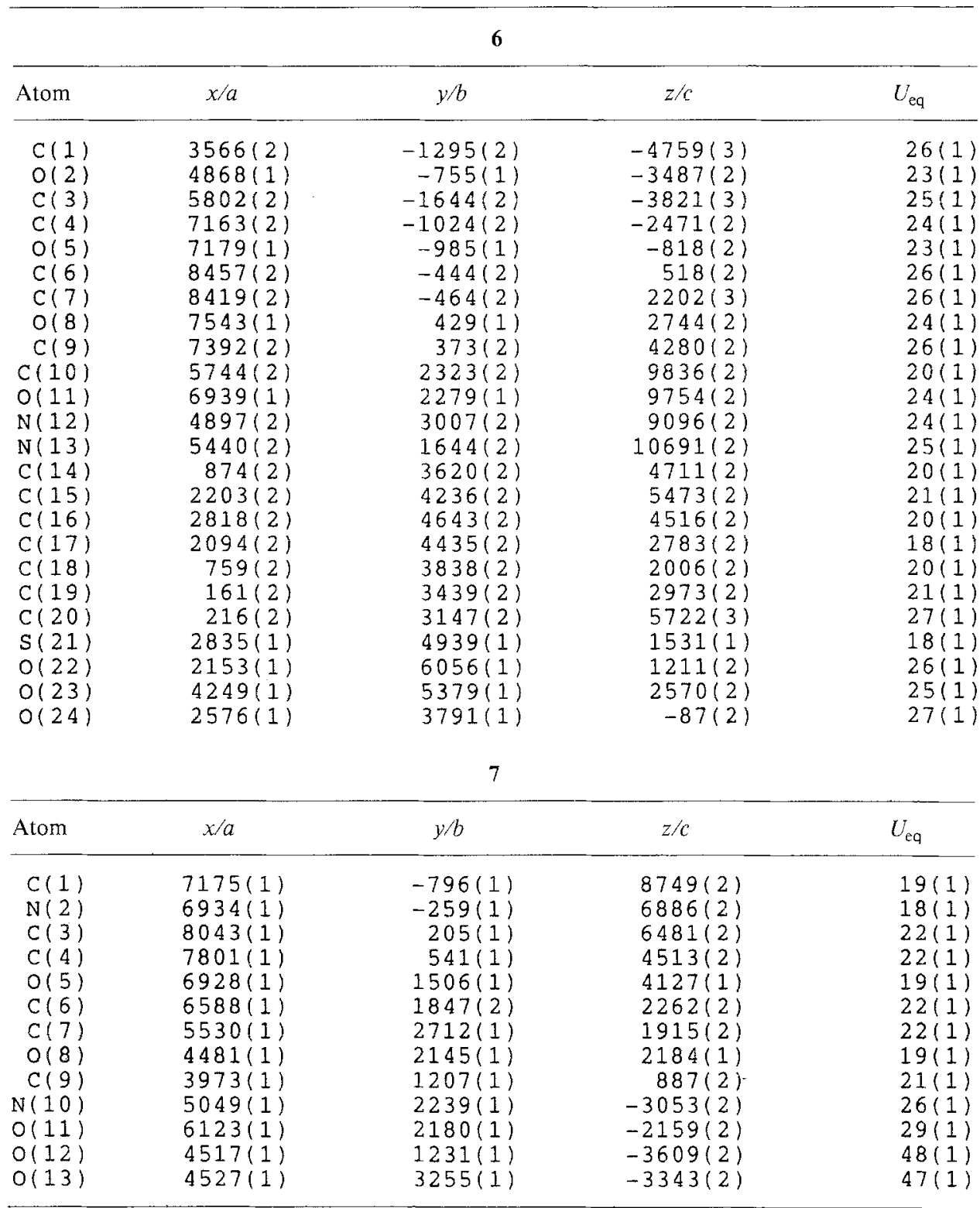

${ }^{a}$ Estimated standard deviations given in parentheses in units of the least significant digit.

b Defined according to Willis and Pryor [29].

c See footnote (a) of Table I. 
Table lll. Torsion angles ${ }^{\text {a }}$ (deg) in the macrocycles of the compounds ${ }^{b}$

\begin{tabular}{lr}
\hline \multicolumn{2}{c}{1} \\
\hline $\mathrm{C}(1)-\mathrm{O}(2)-\mathrm{C}(3)-\mathrm{C}(4)$ & $-176.9(1)$ \\
$\mathrm{O}(2)-\mathrm{C}(3)-\mathrm{C}(4)-\mathrm{O}(5)$ & $66.7(1)$ \\
$\mathrm{C}(3)-\mathrm{C}(4)-\mathrm{O}(5)-\mathrm{C}(6)$ & $179.7(1)$ \\
$\mathrm{C}(4)-\mathrm{O}(5)-\mathrm{C}(6)-\mathrm{C}(7)$ & $170.9(1)$ \\
$\mathrm{O}(5)-\mathrm{C}(6)-\mathrm{C}(7)-\mathrm{O}(8)$ & $64.9(1)$ \\
$\mathrm{C}(6)-\mathrm{C}(7)-\mathrm{O}(8)-\mathrm{C}(9)$ & $172.3(1)$ \\
$\mathrm{C}(7)-\mathrm{O}(8)-\mathrm{C}(9)-\mathrm{C}(1)^{\prime \prime}$ & $170.2(1)$ \\
$\mathrm{O}(8)-\mathrm{C}(9)-\mathrm{C}(1)^{\prime \prime}-\mathrm{O}(2)^{\prime \prime}$ & $-59.3(1)$ \\
$\mathrm{C}(9)-\mathrm{C}(1)^{\prime \prime}-\mathrm{O}(2)^{\prime \prime}-\mathrm{C}(3)^{\prime \prime}$ & $-61.2(1)$ \\
& \\
$\mathrm{C}(10)-\mathrm{O}(11)-\mathrm{C}(12)-\mathrm{C}(13)$ & $-171.1(1)$ \\
$\mathrm{O}(11)-\mathrm{C}(12)-\mathrm{C}(13)-\mathrm{O}(14)$ & $63.2(1)$ \\
$\mathrm{C}(12)-\mathrm{C}(13)-\mathrm{O}(14)-\mathrm{C}(15)$ & $60.4(2)$ \\
$\mathrm{C}(13)-\mathrm{O}(14)-\mathrm{C}(15)-\mathrm{C}(16)$ & $-179.3(1)$ \\
$\mathrm{O}(14)-\mathrm{C}(15)-\mathrm{C}(16)-\mathrm{O}(17)$ & $62.8(1)$ \\
$\mathrm{C}(15)-\mathrm{C}(16)-\mathrm{O}(17)-\mathrm{C}(18)$ & $-178.5(1)$ \\
$\mathrm{C}(16)-\mathrm{O}(17)-\mathrm{C}(18)-\mathrm{C}(10)^{\prime \prime}$ & $173.6(1)$ \\
$\mathrm{O}(17)-\mathrm{C}(18)-\mathrm{C}(10)^{\prime \prime}-\mathrm{O}(11)^{\prime \prime}$ & $62.1(1)$ \\
$\mathrm{C}(18)-\mathrm{C}(10)^{\prime \prime}-\mathrm{O}(11)^{\prime \prime}-\mathrm{C}(12)^{\prime \prime}$ & $173.1(1)$ \\
\hline
\end{tabular}

2

\begin{tabular}{lr}
\hline $\mathrm{C}(1)-\mathrm{O}(2)-\mathrm{C}(3)-\mathrm{C}(4)$ & $-176.1(2)$ \\
$\mathrm{O}(2)-\mathrm{C}(3)-\mathrm{C}(4)-\mathrm{O}(5)$ & $64.9(2)$ \\
$\mathrm{C}(3)-\mathrm{C}(4)-\mathrm{O}(5)-\mathrm{C}(6)$ & $179.3(2)$ \\
$\mathrm{C}(4)-\mathrm{O}(5)-\mathrm{C}(6)-\mathrm{C}(7)$ & $171.9(2)$ \\
$\mathrm{O}(5)-\mathrm{C}(6)-\mathrm{C}(7)-\mathrm{ON}(8)$ & $61.8(2)$ \\
$\mathrm{C}(6)-\mathrm{C}(7)-\mathrm{ON}(8)-\mathrm{C}(9)$ & $168.6(2)$ \\
$\mathrm{C}(7)-\mathrm{ON}(8)-\mathrm{C}(9)-\mathrm{C}(1)^{\prime \prime}$ & $174.6(2)$ \\
$\mathrm{ON}(8)-\mathrm{C}(9)-\mathrm{C}(1)^{\prime \prime}-\mathrm{O}(2)^{\prime \prime}$ & $-56.1(2)$ \\
$\mathrm{C}(9)-\mathrm{C}(1)^{\prime \prime}-\mathrm{O}(2)^{\prime \prime}-\mathrm{C}(3)^{\prime \prime}$ & $-61.7(2)$ \\
& \\
$\mathrm{C}(10)-\mathrm{ON}(11)-\mathrm{C}(12)-\mathrm{C}(13)$ & $-176.3(2)$ \\
$\mathrm{ON}(11)-\mathrm{C}(12)-\mathrm{C}(13)-\mathrm{O}(14)$ & $60.3(2)$ \\
$\mathrm{C}(12)-\mathrm{C}(13)-\mathrm{O}(14)-\mathrm{C}(15)$ & $59.6(2)$ \\
$\mathrm{C}(13)-\mathrm{O}(14)-\mathrm{C}(15)-\mathrm{C}(16)$ & $-178.4(2)$ \\
$\mathrm{O}(14)-\mathrm{C}(15)-\mathrm{C}(16)-\mathrm{O}(17)$ & $61.7(2)$ \\
$\mathrm{C}(15)-\mathrm{C}(16)-\mathrm{O}(17)-\mathrm{C}(18)$ & $-179.8(2)$ \\
$\mathrm{C}(16)-\mathrm{O}(17)-\mathrm{C}(18)-\mathrm{C}(10)^{\prime \prime}$ & $175.1(2)$ \\
$\mathrm{O}(17)-\mathrm{C}(18)-\mathrm{C}(10)^{\prime \prime}-\mathrm{ON}(11)^{\prime \prime}$ & $58.8(2)$ \\
$\mathrm{C}(18)-\mathrm{C}(10)^{\prime \prime}-\mathrm{ON}(11)^{\prime \prime}-\mathrm{C}(12)^{\prime \prime}$ & $169.4(2)$ \\
\end{tabular}

3

$\mathrm{C}(1)-\mathrm{O}(2)-\mathrm{C}(3)-\mathrm{C}(4)$

$169.6(1)$

$66.9(1)$

$\mathrm{O}(2)-\mathrm{C}(3)-\mathrm{C}(4)-\mathrm{O}(5)$

$-170.4(2)$

$\mathrm{C}(3)-\mathrm{C}(4)-\mathrm{O}(5)-\mathrm{C}(6)$
$\mathrm{C}(4)-\mathrm{O}(5)-\mathrm{C}(6)-\mathrm{C}(7)$

$-177.1(1)$

$\mathrm{O}(5)-\mathrm{C}(6)-\mathrm{C}(7)-\mathrm{O}(8)$

$-61.4(1)$

$\mathrm{C}(6)-\mathrm{C}(7)-\mathrm{O}(8)-\mathrm{C}(9)$

$-73.8(2)$

$\mathrm{C}(7)-\mathrm{O}(8)-\mathrm{C}(9)-\mathrm{C}(10)$

$-172.9(1)$

$\mathrm{O}(8)-\mathrm{C}(9)-\mathrm{C}(10)-\mathrm{O}(11)$

$-72.4(1)$

172.8(2)

$176.5(1)$

$76.1(3)$

$177.9(2)$

$72.2(4)$

$61.8(4)$

$-174.5(5)$

$-179.3(2)$

$-67.3(3)$

$173.6(5)$

$\mathrm{O}(11)-\mathrm{C}(12)-\mathrm{C}(13)-\mathrm{O}\left(14^{\prime}\right)$

$59.5(8)$

$\mathrm{C}(12)-\mathrm{C}(13)-\mathrm{O}\left(14^{\prime}\right)-\mathrm{C}\left(15^{\prime}\right)$

$\mathrm{C}(13)-\mathrm{O}\left(14^{\prime}\right)-\mathrm{C}\left(15^{\prime}\right)-\mathrm{C}\left(16^{\prime}\right)$

$\mathrm{O}\left(14^{\prime}\right)-\mathrm{C}\left(15^{\prime}\right)-\mathrm{C}\left(16^{\prime}\right)-\mathrm{O}\left(17^{\prime}\right)$

$\mathrm{C}\left(15^{\prime}\right)-\mathrm{C}\left(16^{\prime}\right)-\mathrm{O}\left(17^{\prime}\right)-\mathrm{C}\left(18^{\prime}\right)$

$\mathrm{C}\left(16^{\prime}\right)-\mathrm{O}\left(17^{\prime}\right)-\mathrm{C}\left(18^{\prime}\right)-\mathrm{C}(1)$

$\mathrm{O}\left(17^{\prime}\right)-\mathrm{C}\left(18^{\prime}\right)-\mathrm{C}(1)-\mathrm{O}(2)$

$\mathrm{C}\left(18^{\prime}\right)-\mathrm{C}(1)-\mathrm{O}(2)-\mathrm{C}(3)$

$-175.1(5)$

$176.8(8)$

$-61.2(9)$

$-66(1)$

$-175.0(5)$

$-73.7(6)$

$158(1)$

\begin{tabular}{lr}
\hline \\
\hline$C(1)-O(2)-C(3)-C(4)$ & $82.7(2)$ \\
$O(2)-C(3)-C(4)-O(5)$ & $62.0(2)$ \\
$C(3)-C(4)-O(5)-C(6)$ & $176.2(2)$ \\
$C(4)-O(5)-C(6)-C(7)$ & $178.9(2)$ \\
$O(5)-C(6)-C(7)-O(8)$ & $-74.2(1)$ \\
$\mathrm{C}(6)-\mathrm{C}(7)-\mathrm{O}(8)-\mathrm{C}(9)$ & $-175.1(2)$ \\
$\mathrm{C}(7)-\mathrm{O}(8)-\mathrm{C}(9)-\mathrm{C}(10)$ & $-176.7(1)$ \\
$\mathrm{O}(8)-\mathrm{C}(9)-\mathrm{C}(10)-\mathrm{O}(11)$ & $74.5(1)$ \\
$\mathrm{C}(9)-\mathrm{C}(10)-\mathrm{O}(11)-\mathrm{C}(12)$ & $-178.5(2)$ \\
$\mathrm{C}(10)-\mathrm{O}(11)-\mathrm{C}(12)-\mathrm{C}(13)$ & $82.6(2)$ \\
$\mathrm{O}(11)-\mathrm{C}(12)-\mathrm{C}(13)-\mathrm{O}(14)$ & $62.5(2)$ \\
$\mathrm{C}(12)-\mathrm{C}(13)-\mathrm{O}(14)-\mathrm{C}(15)$ & $174.1(2)$ \\
$\mathrm{C}(13)-\mathrm{O}(14)-\mathrm{C}(15)-\mathrm{C}(16)$ & $177.5(2)$ \\
$\mathrm{O}(14)-\mathrm{C}(15)-\mathrm{C}(16)-\mathrm{O}(17)$ & $-72.6(2)$ \\
$\mathrm{C}(15)-\mathrm{C}(16)-\mathrm{O}(17)-\mathrm{C}(18)$ & $-172.7(2)$ \\
$\mathrm{C}(16)-\mathrm{O}(17)-\mathrm{C}(18)-\mathrm{C}(1)$ & $-174.6(2)$ \\
$\mathrm{O}(17)-\mathrm{C}(18)-\mathrm{C}(1)-\mathrm{O}(2)$ & $72.8(1)$ \\
$\mathrm{C}(18)-\mathrm{C}(1)-\mathrm{O}(2)-\mathrm{C}(3)$ & $-176.4(2)$ \\
\hline
\end{tabular}

\section{5}

$C(1)-O(2)-C(3)-C(4)$
$O(2)-C(3)-C(4)-O(5)$
$C(3)-C(4)-O(5)-C(6)$
$C(4)-O(5)-C(6)-C(7)$
$O(5)-C(6)-C(7)-O(8)$
$C(6)-C(7)-O(8)-C(9)$
$C(7)-O(8)-C(9)-C(10)$
$O(8)-C(9)-C(10)-O(11)$
$C(9)-C(10)-O(11)-C(12)$
$C(10)-O(11)-C(12)-C(13)$
$O(11)-C(12)-C(13)-O(14)$
$C(12)-C(13)-O(14)-C(15)$

$76.4(2)$

$63.7(2)$

$175.7(2)$

$-172.7(2)$

$-73.0(2)$

$-178.9(2)$

$-174.9(2)$

$68.9(2)$

$-173.7(2)$

$83.2(2)$

$70.4(2)$

$176.0(2)$ 


\begin{tabular}{|c|c|c|c|}
\hline$C(13)-O(14)-C(15)-C(16)$ & $174.5(2)$ & \multicolumn{2}{|l|}{7} \\
\hline$O(14)-C(15)-C(16)-O(17)$ & $-71.9(2)$ & & \multirow{7}{*}{$\begin{array}{r}170.8(1) \\
60.5(1) \\
-176.9(1) \\
170.9(1) \\
-64.6(1) \\
-68.1(1) \\
-174.8(1)\end{array}$} \\
\hline$C(15)-C(16)-O(17)-C(18)$ & $-176.2(3)$ & \multirow{7}{*}{$\begin{array}{l}\mathrm{C}(1)-\mathrm{N}(2)-\mathrm{C}(3)-\mathrm{C}(4) \\
\mathrm{N}(2)-\mathrm{C}(3)-\mathrm{C}(4)-\mathrm{O}(5) \\
\mathrm{C}(3)-\mathrm{C}(4)-\mathrm{O}(5)-\mathrm{C}(6) \\
\mathrm{C}(4)-\mathrm{O}(5)-\mathrm{C}(6)-\mathrm{C}(7) \\
\mathrm{O}(5)-\mathrm{C}(6)-\mathrm{C}(7)-\mathrm{O}(8) \\
\mathrm{C}(6)-\mathrm{C}(7)-\mathrm{O}(8)-\mathrm{C}(9) \\
\mathrm{C}(7)-\mathrm{O}(8)-\mathrm{C}(9)-\mathrm{C}(1)^{\prime \prime} \\
\mathrm{O}(8)-\mathrm{C}(9)-\mathrm{C}(1)^{\prime \prime}-\mathrm{N}(2)^{\prime \prime} \\
\mathrm{C}(9)-\mathrm{C}(1)^{\prime \prime}-\mathrm{N}(2)^{\prime \prime}-\mathrm{C}(3)^{\prime \prime}\end{array}$} & \\
\hline$C(16)-O(17)-C(18)-C(1)$ & $-173.4(2)$ & & \\
\hline$O(17)-C(18)-C(1)-O(2)$ & $71.0(2)$ & & \\
\hline$C(18)-C(1)-O(2)-C(3)$ & $-175.1(2)$ & & \\
\hline \multicolumn{2}{|l|}{6} & & \\
\hline$C(1)-O(2)-C(3)-C(4)$ & $178.9(1)$ & & \\
\hline$O(2)-C(3)-C(4)-O(5)$ & $68.7(1)$ & & $-178.0(1)$ \\
\hline$C(3)-C(4)-O(5)-C(6)$ & $178.2(2)$ & & \\
\hline$C(4)-O(5)-C(6)-C(7)$ & $-178.5(1)$ & \multirow{6}{*}{$\begin{array}{l}\text { a See footnote (a) of Table II. } \\
\text { b See footnote (a) of Table I. }\end{array}$} & \\
\hline$O(5)-C(6)-C(7)-O(8)$ & $-65.6(1)$ & & \\
\hline$C(6)-C(7)-O(8)-C(9)$ & $175.7(1)$ & & \\
\hline$C(7)-O(8)-C(9)-C(1)^{\prime \prime}$ & $-178.3(1)$ & & \\
\hline $\mathrm{O}(8)-\mathrm{C}(9)-\mathrm{C}(1)^{\prime \prime}-\mathrm{O}(2)^{\prime \prime}$ & $69.5(1)$ & & \\
\hline$C(9)-C(1)^{\prime \prime}-O(2)^{\prime \prime}-C(3)^{\prime \prime}$ & $-176.0(1)$ & & \\
\hline
\end{tabular}

The crystal structure of the $1: 5$ adduct of 18 -crown- 6 with urea (1) is best explained as consisting of layers of 18 -crown-6 molecules alternating with layers of urea molecules (Figure 1). The asymmetric unit consists of two half 18 -crown-6 molecules (the other halves are generated by a centre of symmetry) and five urea molecules, of which three (with central C-atoms 27,31 and 35) are forming the urea layer, held together by a 2-dimensional hydrogen bonding network. The remaining two urea molecules (central C-atoms 19 and 23) form links between both layers, each of them forming two hydrogen bonds to an 18-crown-6 molecule (and thus forming an 18crown-6: urea complex with a $1: 2$ ratio) and two to the urea layer. The urea oxygen atoms $\mathrm{O}(20)$ and $\mathrm{O}(24)$ accept four and three hydrogen bonds resp. from the urea layer. All urea hydrogens are used in hydrogen bonding (see Table IV). Because both crown units accept two hydrogen bonds at neighbouring oxygen atoms at each side of the ring, this can be denoted as a 'double 1,2' hydrogen-bond scheme. Although the two crown units are different from the crystallographic point of view, the conformations of both are the same (the 'biangular' conformation with torsion code $g^{+} g^{+} a a g^{-} a$ $a g^{-} a g^{-} g^{-} a \operatorname{ag}^{+} a \operatorname{ag}^{+} a$, see Table III).

The 1:5 adduct of aza-18-crown-6 and urea (complex 2) is isostructural with complex 1. This implies disordering because the aza-18-crown-6 ring is not centrosymmetric. The ring nitrogen atom can occupy two positions related by a centre of symmetry. Mean deviations in positions between corresponding atoms in complexes 1 and 2 are only $0.044 \AA$ for non-hydrogen atoms and $0.077 \AA$ for hydrogens. The deviations between non-hydrogen atoms are largest for atoms $O(2)$ and $O(11)$ in complex 1 vs atoms $\mathrm{ON}(2)$ and $\mathrm{ON}(11)$ in complex 2 (being the average of half an oxygen and half a nitrogen atom) and for atoms close to these. The ring nitrogens are used as acceptors for hydrogen bonds (see Figure 2).

The hydrogen bonding scheme in the $1: 1$ complex of $O$ - $n$-butylisouronium picrate with 18-crown-6 (3) is different from the previous ones (Figure 3a). Three of the four amino hydrogens are hydrogen bonded to the crown ether, while one hydrogen bond is formed between the fourth hydrogen and the picrate anion. By analogy 
Table IV. Hydrogen-Bond parameters ${ }^{\mathrm{a}}$ for the compounds ${ }^{\mathrm{b}}$

\begin{tabular}{|c|c|c|c|c|c|c|c|c|c|}
\hline $\begin{array}{l}\text { Donor } \\
\text { atom: D }\end{array}$ & $\begin{array}{l}\text { Accep- } \\
\text { tor } \\
\text { atom: A }\end{array}$ & $\begin{array}{l}\text { Distance } \\
\mathrm{D} \cdots \mathrm{A} \\
(\AA)\end{array}$ & $\begin{array}{l}\text { Distance } \\
\mathrm{H} \cdots \mathrm{A} \\
(\AA)\end{array}$ & $\begin{array}{l}\text { Angle } \\
\mathrm{D}-\mathrm{H} \cdots \mathrm{A} \\
(\mathrm{deg})\end{array}$ & $\begin{array}{l}\text { Donor } \\
\text { atom: D }\end{array}$ & $\begin{array}{l}\text { Accep- } \\
\text { tor } \\
\text { atom: A }\end{array}$ & $\begin{array}{l}\text { Distance } \\
\text { D } \cdots A \\
(\AA)\end{array}$ & $\begin{array}{l}\text { Distance } \\
\mathrm{H} \cdots \mathrm{A} \\
(\AA)\end{array}$ & $\begin{array}{l}\text { Angle } \\
\text { D-H } \cdots A \\
(\text { deg) }\end{array}$ \\
\hline \multicolumn{5}{|c|}{1} & \multicolumn{5}{|c|}{3} \\
\hline$N(21)$ & $O(28)$ & $2.912(2)$ & $1.98(2)$ & $172(2)$ & $N(21)$ & $O(8)$ & $2.888(2)$ & $2.08(2)$ & $161(2)$ \\
\hline$N(21)$ & $O(2)$ & $2.848(2)$ & $2.04(2)$ & $174(2)$ & $N(21)$ & $\mathrm{O}(33)$ & $2.839(2)$ & $2.01(2)$ & $172(3)$ \\
\hline$N(22)$ & $O(8)$ & $3.282(2)$ & $2.55(2)$ & $154(2)$ & $N(22)$ & $\mathrm{O}(5)$ & $3.003(2)$ & $2.29(2)$ & $136(2)$ \\
\hline$N(22)$ & $O(36)$ & $3.057(2)$ & $2.18(2)$ & $165(2)$ & $N(22)$ & $O(8)$ & $3.218(2)$ & $2.46(2)$ & $141(2)$ \\
\hline$N(25)$ & $\mathrm{O}(32)$ & $3.017(2)$ & $2.13(2)$ & $167(2)$ & $N(22)$ & $O(17)$ & $2.997(4)$ & $2.16(3)$ & $164(2)$ \\
\hline$N(25)$ & $O(11)$ & $3.214(2)$ & $2.46(2)$ & $150(2)$ & $\mathrm{N}(22)$ & $O\left(17^{\prime}\right)$ & $2.842(8)$ & $1.98(3)$ & $172(4)$ \\
\hline$N(26)$ & $O(14)$ & $2.893(2)$ & $2.07(2)$ & $163(2)$ & & & & & \\
\hline$N(26)$ & $O(28)$ & $2.932(2)$ & $2.05(2)$ & $177(2)$ & \multicolumn{5}{|c|}{4} \\
\hline$N(29)$ & $O(20)$ & $3.030(2)$ & $2.13(2)$ & $175(2)$ & & & & & \\
\hline$N(29)$ & $O(32)$ & $2.913(2)$ & $2.12(2)$ & $158(2)$ & $O(20)$ & $O(24)$ & $2.482(2)$ & $1.54(3)$ & $166(3)$ \\
\hline$N(30)$ & $O(24)$ & $2.884(2)$ & $2.00(2)$ & $173(2)$ & $N(21)$ & $O(5)$ & $3.177(2)$ & $2.37(3)$ & $142(2)$ \\
\hline$N(30)$ & $O(24)$ & $2.922(2)$ & $2.08(2)$ & $173(2)$ & $N(21)$ & $O(11)$ & $2.932(2)$ & $2.08(3)$ & $168(3)$ \\
\hline$N(33)$ & $O(36)$ & $3.018(2)$ & $2.16(2)$ & $169(2)$ & $N(22)$ & $O(2)$ & $2.839(2)$ & $1.98(3)$ & $168(3)$ \\
\hline$N(33)$ & $O(20)$ & $3.056(2)$ & $2.26(2)$ & $153(2)$ & $N(22)$ & $O(25)$ & $3.091(2)$ & $2.23(3)$ & $177(3)$ \\
\hline$N(34)$ & $O(28)$ & $2.889(2)$ & $2.07(2)$ & $172(2)$ & & & & & \\
\hline$N(34)$ & $O(24)$ & $2.922(2)$ & $2.07(2)$ & $171(2)$ & \multicolumn{5}{|c|}{5} \\
\hline$N(37)$ & $O(20)$ & $2.93 !(2)$ & $2.07(2)$ & $172(2)$ & & & & & \\
\hline$N(37)$ & $O(36)$ & $2.925(2)$ & $2.10(2)$ & $160(2)$ & $O(20)$ & $O(29)$ & $2.560(3)$ & $1.73(4)$ & $165(4)$ \\
\hline$N(38)$ & $O(20)$ & $2.985(2)$ & $2.14(2)$ & $167(2)$ & $N(2 I)$ & $O(2)$ & $2.801(3)$ & $1.89(4)$ & $169(4)$ \\
\hline$N(38)$ & $O(32)$ & $2.920(2)$ & $2.08(2)$ & $173(1)$ & $N(21)$ & $O(29)$ & $2.751(3)$ & $2.06(3)$ & $141(3)$ \\
\hline & & & & & $N(21)$ & $\mathrm{O}(31)$ & $3.031(4)$ & $2.32(3)$ & $145(3)$ \\
\hline \multicolumn{5}{|c|}{2} & $N(22)$ & $\mathrm{O}(5)$ & $2.995(3)$ & $2.23(3)$ & $152(3)$ \\
\hline & & & & & $N(22)$ & $\mathrm{O}(11)$ & $2.828(3)$ & $1.94(3)$ & $170(3)$ \\
\hline$N(21)$ & $\mathrm{O}(28)$ & $2.923(2)$ & $2.01(2)$ & $171(2)$ & & & & & \\
\hline$N(21)$ & $O(2)$ & $2.858(2)$ & $1.95(2)$ & $179(2)$ & \multicolumn{5}{|c|}{6} \\
\hline$N(22)$ & $O N(8)$ & $3.273(2)$ & $2.43(3)$ & $164(2)$ & & & & & \\
\hline $\mathrm{N}(22)$ & $O(36)$ & $3.034(2)$ & $2.19(3)$ & $167(2)$ & $O(11)$ & $\mathrm{O}(22)$ & $2.531(2)$ & $1.59(3)$ & $169(3)$ \\
\hline$N(25)$ & $O(32)$ & $3.004(2)$ & $2.07(3)$ & $171(2)$ & $N(12)$ & $O(23)$ & $2.972(2)$ & $2.00(3)$ & $178(3)$ \\
\hline$N(25)$ & ON(11) & $3.217(3)$ & $2.39(2)$ & $162(2)$ & $N(12)$ & $O(24)$ & $2.870(2)$ & $2.02(3)$ & $174(3)$ \\
\hline$N(26)$ & $\mathrm{O}(14)$ & $2.898(2)$ & $2.00(3)$ & $165(2)$ & $N(13)$ & $O(5)$ & $2.936(2)$ & $2.07(3)$ & $166(3)$ \\
\hline$N(26)$ & $O(28)$ & $2.945(2)$ & $2.05(3)$ & $177(2)$ & $N(13)$ & $\mathrm{O}(8)$ & $2.996(2)$ & $2.12(2)$ & $173(3)$ \\
\hline$N(29)$ & $\mathrm{O}(20)$ & $3.028(2)$ & $2.14(3)$ & $171(2)$ & & & & & \\
\hline$N(29)$ & $O(32)$ & $2.909(2)$ & $2.15(2)$ & $157(2)$ & \multicolumn{5}{|c|}{7} \\
\hline$N(30)$ & $O(24)$ & $2.884(2)$ & $2.02(2)$ & $174(2)$ & & & & & \\
\hline$N(30)$ & $O(24)$ & $2.921(2)$ & $2.04(3)$ & $177(2)$ & $N(2)$ & $O(11)$ & $2.926(2)$ & $2.12(2)$ & $148(2)$ \\
\hline$N(33)$ & $O(36)$ & $3.026(2)$ & $2.15(3)$ & $170(2)$ & $N(2)$ & $O(12)$ & $3.166(2)$ & $2.33(2)$ & $153(2)$ \\
\hline$N(33)$ & $O(20)$ & $3.041(2)$ & $2.20(3)$ & $156(2)$ & $N(2)^{\prime \prime}$ & $O(12)$ & $2.810(2)$ & $2.00(2)$ & $150(2)$ \\
\hline$N(34)$ & $O(28)$ & $2.894(2)$ & $2,07(3)$ & $169(2)$ & $N(2)^{\prime \prime}$ & $O(8)$ & $2.813(2)$ & $2.49(2)$ & $102(1)$ \\
\hline$N(34)$ & $O(24)$ & $2.928(2)$ & $2.04(3)$ & $173(2)$ & \multirow{5}{*}{\multicolumn{5}{|c|}{$\begin{array}{l}\text { a See footnote (a) of Table I. } \\
\text { b See footnote (a) of Table I. }\end{array}$}} \\
\hline$N(37)$ & $O(20)$ & $2.942(2)$ & $2.07(2)$ & $171(2)$ & & & & & \\
\hline$N(37)$ & $O(36)$ & $2.920(2)$ & $2.13(2)$ & $163(2)$ & & & & & \\
\hline$N(38)$ & $O(20)$ & $2.972(2)$ & $2.15(2)$ & $168(2)$ & & & & & \\
\hline$N(38)$ & $O(32)$ & $2.921(2)$ & $2.05(2)$ & $170(2)$ & & & & & \\
\hline
\end{tabular}



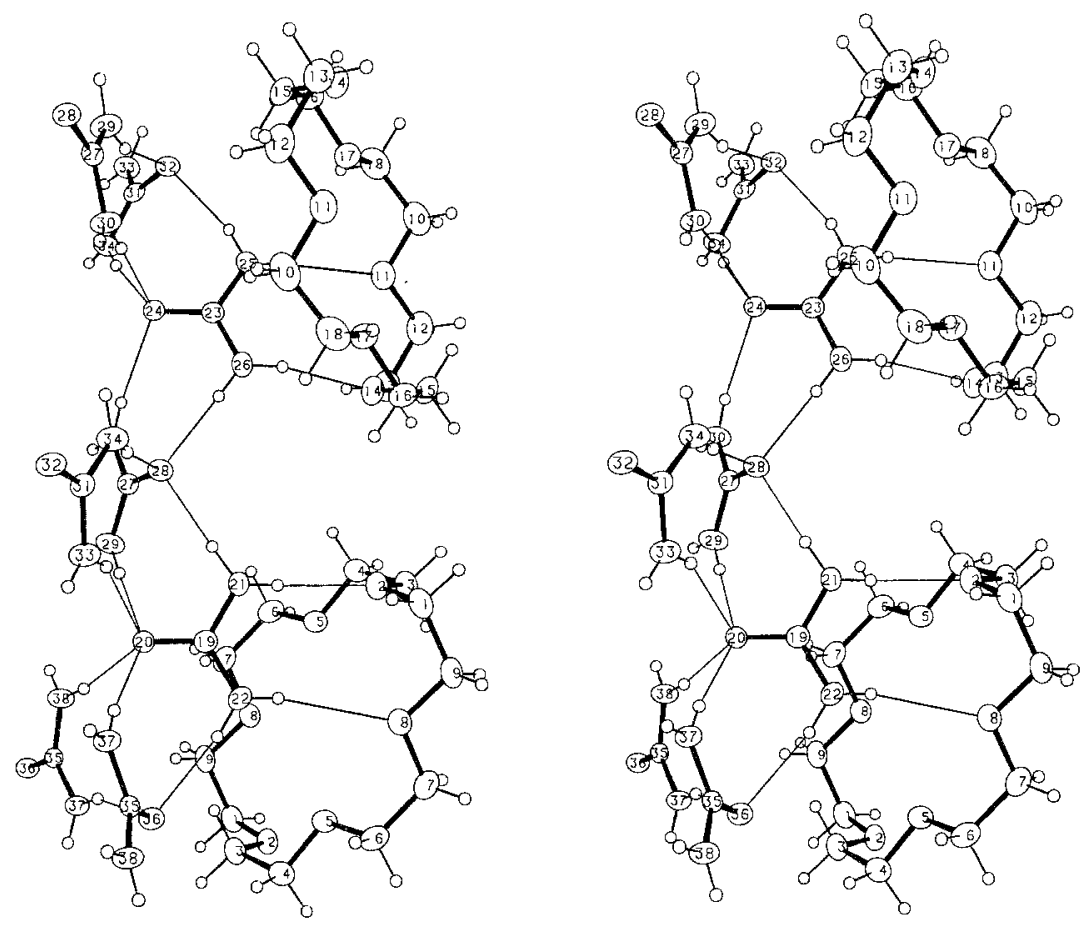

Fig. 1. Stereoview of the 18-crown-6 (urea) 5 complex (1) showing the atom numbering. Atoms with equal numbers are symmetry-related. Only the environment for one side of the centrosymmetric macrorings is shown.
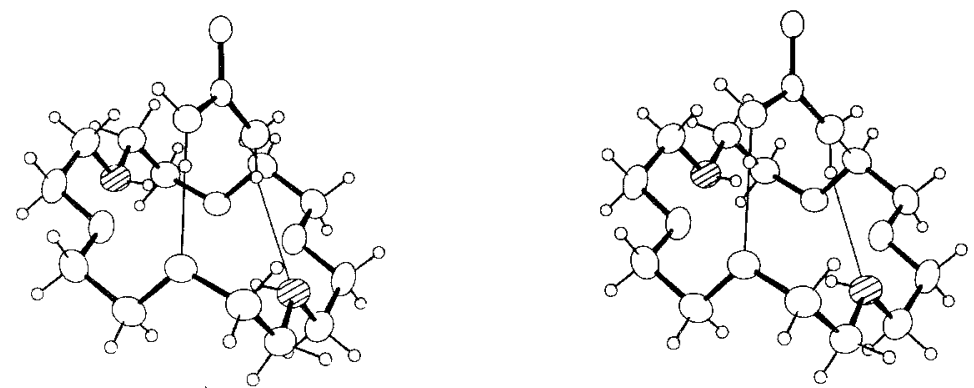

Fig. 2. Stereoview of one macroring and one hydrogen-bonded guest molecule of the aza-18-crown-6 . (urea) $)_{5}$ complex (2). The urea molecule at the bottom side is not shown. Polyether nitrogen/oxygen atom positions are indicated. The atom numbering is according to the numbering in the isostructural complex 1.

with complexes $\mathbf{1}$ and $\mathbf{2}$, the bonding between cation and crown can be denoted as 'single 1,2,4' complexation. As explained in the experimental section, the crystal structure is composed of a mixture of $72.1 \%$ molecules with the spatial arrangement as depicted in Figure 3a (macroring with torsion code $g^{+} g^{+} a a^{-} a \mathrm{ag}^{+} a \mathrm{ag}^{-} \mathrm{g}^{-}$ $a g^{-} a a^{+} a$ ), and $27.9 \%$ molecules with a slightly different conformation of the macrocycle (torsion code $g^{+} g^{+} a a g^{-} a a^{+} a g^{+} g^{+} a a g^{-} a a g^{+} a$ ). Both conformations of the ring, including the hydrogen bonds to the cation are depicted in Figure $3 \mathrm{~b}$. The atoms with different coordinates in both conformations are denoted without prime for the 

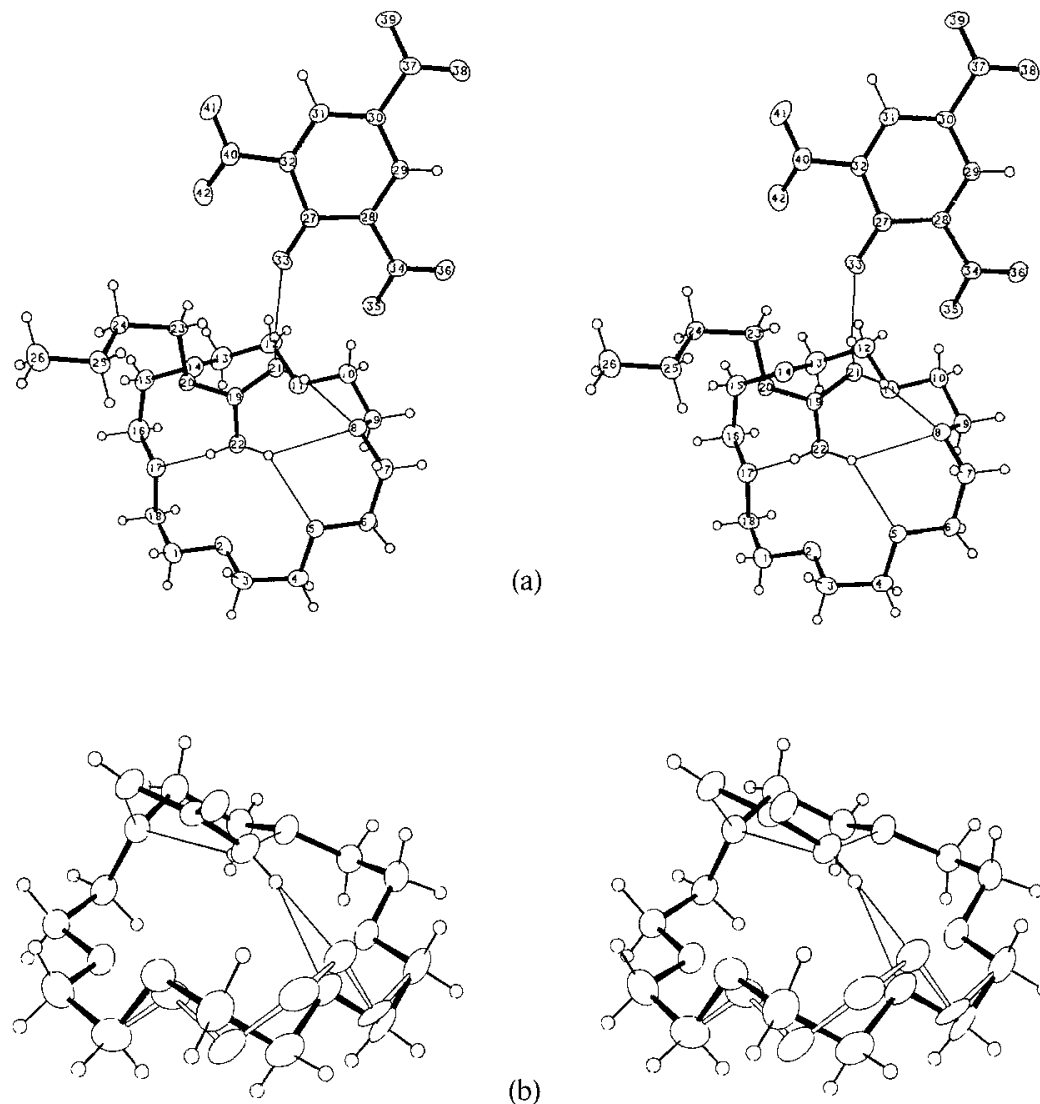

(b)

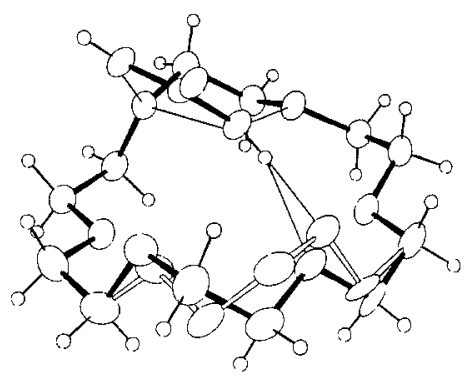

Fig. 3. (a): stereoview of the 18-crown-6.O-n-butylisouronium picrate complex (3) in the predominant conformation, showing the atom numbering. (b): stereoview of the two partly occupied conformations of the macroring foccupation 72.1\%: solid lines; occupation $27.9 \%$; open lines). For the sake of clarity only the $\mathrm{OC}\left(\mathrm{NH}_{2}\right)_{2}$-part of the cation is shown.

highest occupied and with prime for the lowest occupied conformation in Tables II-IV (atoms $\mathrm{O}(14)-\mathrm{C}(18)$ and $\mathrm{O}\left(14^{\prime}\right)-\mathrm{C}\left(18^{\prime}\right)$, resp.).

The X-ray analysis of the $1: 1$ complex between 18-crown-6 and uronium nitrate (4) shows that this complex has the same hydrogen bonding scheme as found for complex 3 (Figure 4). Three of the four amine hydrogens are hydrogen bonded to the macroring ('single $1,2,4$ ' complexation) and the fourth to the anion. The hydrogen at the oxygen of the uronium ion is involved in a short bond $(2.482 \AA)$ to a second nitrate anion. The macroring has the same conformation as the lowest-occupied conformation in complex 3 (torsion code $g^{+} g^{+} a a g^{-} a a^{+} a g^{+} g^{+} a a^{-} a g^{+} a$ ).

The structure of the $1: 1$ complex of 18-crown-6 with uronium picrate (5) reveals the same complexation characteristics as found in complex $\mathbf{4}$, i.e., 'single $1,2,4$ ' complexation between cation and crown, whereas the fourth amino hydrogen and the hydrogen at the oxygen are used in hydrogen bonding with the anion (Figure 5). The conformation of the crown is also identical to the one in complex 4 (torsion code $g^{+} g^{+} a a g^{-} a a g^{+} a g^{+} g^{+} a a g^{-} a a g^{+} a$ ). Therefore, the choice of anion seems to have little influence on the complexational and conformational properties of the crown-cation moiety. 

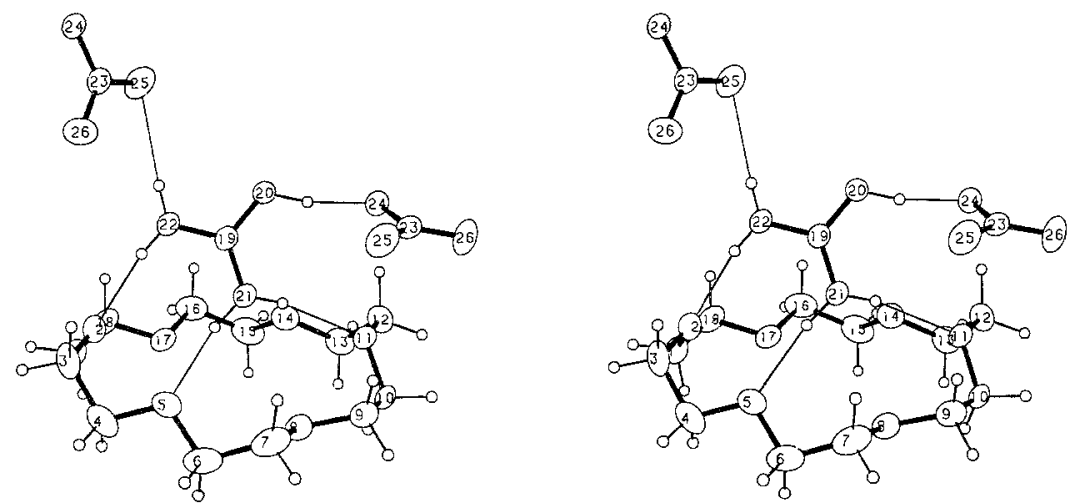

Fig. 4. Stereoview of the 18 -crown-6.uronium nitrate complex (4) showing the atom numbering. The two anions are symmetry-related.
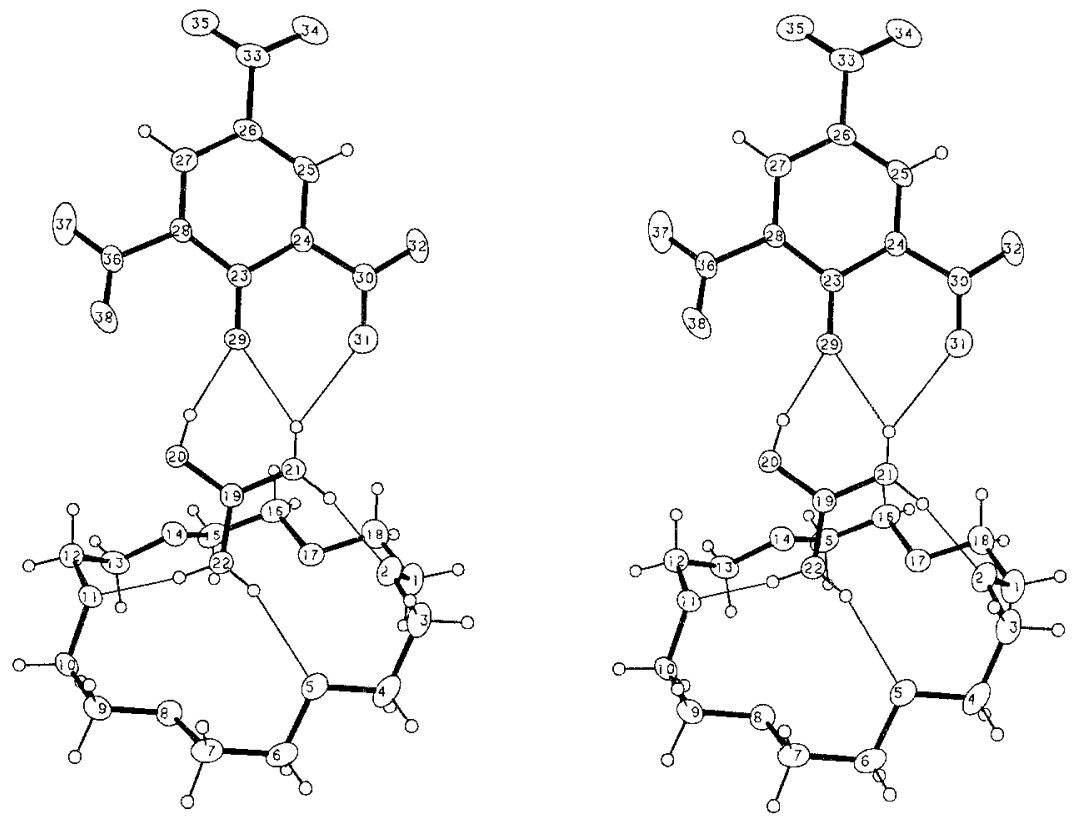

Fig. 5. Stereoview of the 18-crown-6 t uronium picrate complex (5) showing the atom numbering.

That affairs are not as simple, however, is shown by the complexation between 18-crown-6 and uronium $p$-toluenesulphonate (6), as not only the conformation adopted and the hydrogen bonding scheme involved are different, but even the stoichiometry changes to a $1: 2$ ratio (see Figure 6). The asymmetric unit consists of one half crown unit (the other half is generated by a centre of symmetry), with one guest molecule. Only one $-\mathrm{NH}_{2}$ group per uronium ion is hydrogen bonded to the crown, to next-nearest neighbour oxygen atoms ('double 1,3' complexation). The other three uronium hydrogens form hydrogen bonds to the anions, including again a short $\mathrm{O}-\mathrm{H} \cdots \mathrm{O}$ contact $(2.531 \AA)$. The crown ether molecule has the well-known conformation with almost $D_{3 \mathrm{~d}}$ symmetry (torsion code $a g^{+} a \mathrm{ag}^{-} a \mathrm{ag}^{+} a \mathrm{ag}^{-} a \mathrm{ag}^{+} a$ $a g^{-} a$ ). The same complexation scheme and the same $D_{3 \mathrm{~d}}$ conformation are also 

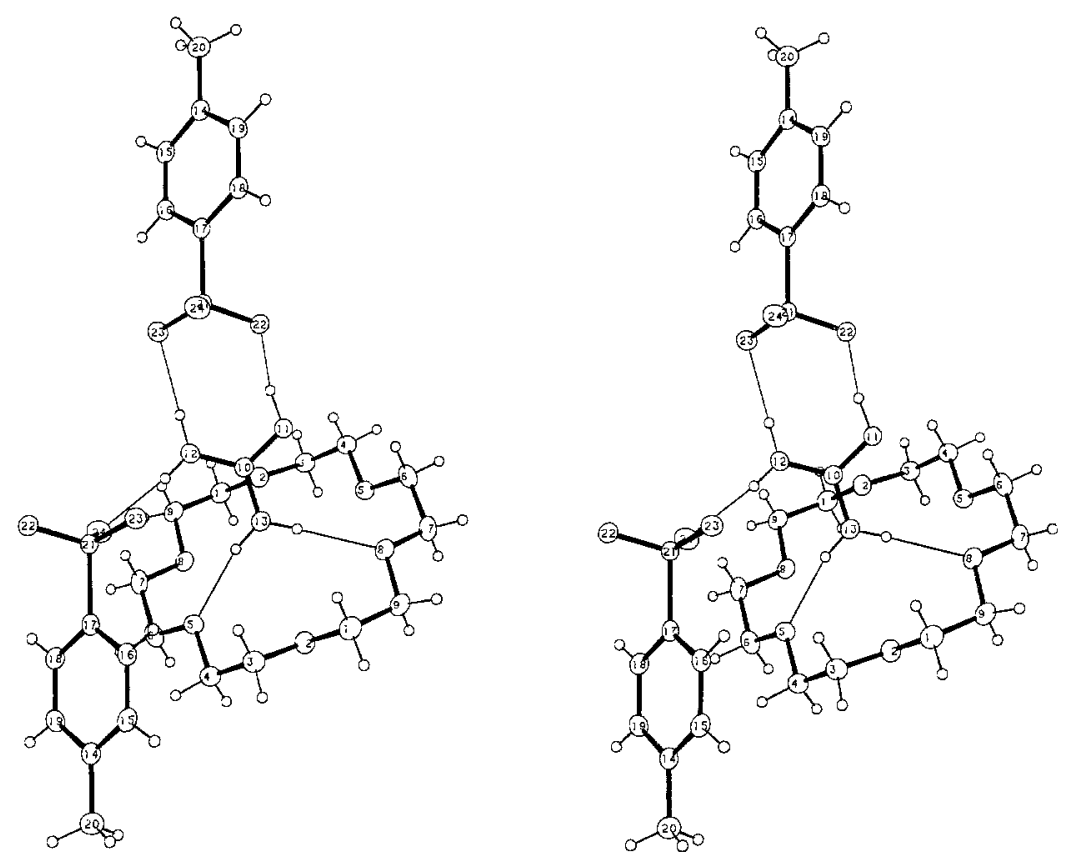

Fig. 6. Stereoview of the 18-crown-6.(uronium $p$-toluenesulphonate) 2 complex (6) showing the atom numbering. Only the environment at one side of the centrosymmetric macroring is shown. The two anions are symmetry-related.

found for the $1: 2$ complex between 18-crown-6 and S-tert-butylisothiouronium perchlorate [25].

To study the influence of amino groups in the polyether rings on complexation of uronium ions with crown ethers, a complex analogous to complex 4 was planned, using diaza-18-crown-6 as polyether. X-Ray analysis revealed, however, that the resulting solid consists of the dinitrate salt of the diprotonated macroring (compound 7). This means that the uronium cation dissociates on complexation, thereby protonating the host. Whereas the urea molecules formed are not part of the complex precipitated, the nitrate anions are, via three hydrogen bonds per anion to two amine hydrogen atoms of the host (see Figure 7). As the hydrogen bonds are donated from the host instead of from the guest, the resulting hydrogen-bonding scheme can be described as an 'inverted'-double-1,4 complexation. The conformation adopted by the macroring is again the "biangular" $g^{+} g^{+} a a g^{-} a a^{-} a g^{-} g^{-} a a g^{+} a a g^{+} a$ conformation. It is assumed that this conformation is favoured over the $D_{3 \mathrm{~d}}$ conformation, adopted by the free diaza-18-crown-6 molecule [30], because in the "biangular" conformation all four amine hydrogens are equally directed to points above and below the macrocycle, thus being able to form hydrogen bonds to two nitrate anions (Figure 7). In the $D_{3 \mathrm{~d}}$ conformation this would be impossible, as one amine hydrogen per nitrogen atom would be directed almost to the centre of the ring and the other almost perpendicular to it. Each amine hydrogen is not only involved in an intermolecular hydrogen bond to a nitrate anion, but also in an intramolecular 'hydrogen bond' with a neighbouring oxygen atom (not drawn in Figure 7). 

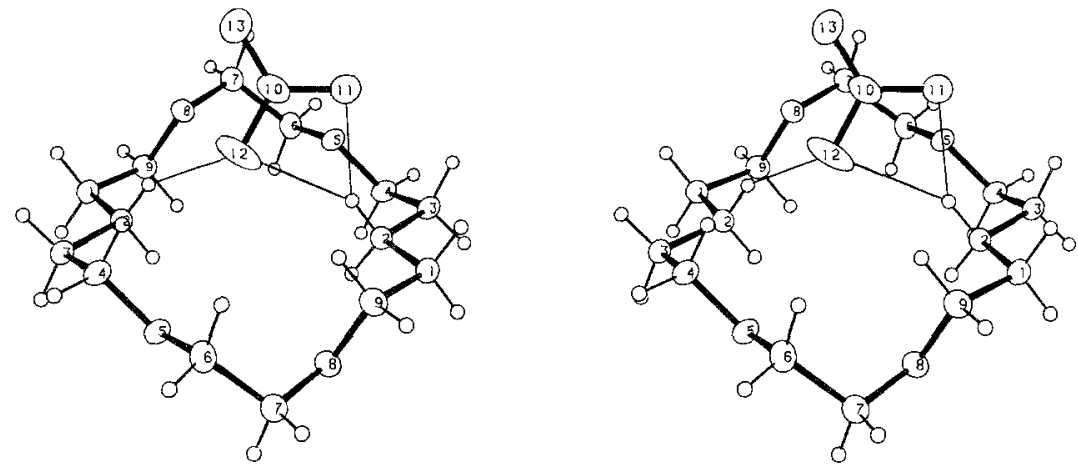

Fig. 7. Stereoview of the diaza-18-crown-6 $\left(\mathrm{HNO}_{3}\right)_{2}$ compound (7) showing the atom numbering. Only the environment at one side of the centrosymmetric macroring is shown.

\section{Discussion}

\subsection{COMPLEXATION SCHEMES}

(Aza-) 18-crown-6 is able to form different types of complexes with similar guests. Both $1: 2$ and 1:1 host/guest adducts are possible and the adducts formed may or may not be separated by interspatial layers of guest molecules, not bonded to host molecules.

A literature survey shows the same trends. Recently, some X-ray structure determinations of complexes of (diaza-) 18-crown-6 with neutral urea-like guests have been reported [6a-6e, 3e]. A similar division according to complexation type among these complexes can be made. Firstly, the $1: 4$ complexes of 18-crown-6 [6a] and of diaza-18-crown-6 [6e] with thiourea are both comparable with the structures of complexes 1 and 2: the polyethers form $1: 2$ adducts with the guest molecules via a similar hydrogen-bonding scheme as above (in the diaza-complex, the nitrogen atoms are used as hydrogen-bond acceptors, like in complex 2 ), whereas the remaining thiourea molecules form layers in between, held together by hydrogen-bond bridges. Secondly, 18-crown-6 forms solid $1: 2$ complexes with thiourea [6b], N,N'-dimethylthiourea [6c] and with $m$-chlorophenylurea [6d], with a $1: 2$ polyether/guest ratio, without interadjacent layers. Additionally, 18-crown-6 can form a $1: 1$ complex with $\mathrm{N}$-methylthiourea [3e] in the crystalline state. However, effectively the polyether also forms a $1: 2$ adduct with the guests, as each guest bridges between two macrorings, thus forming infinitely long chains.

Surprisingly, the complex formed between 18 -crown- 6 and guanidinium nitrate, as reported by Truter and coworkers [26], has a structure completely different from the analogous complex between 18-crown-6 and uronium nitrate (complex 4). Firstly, the guanidinium nitrate forms a $2: 1$ complex with 18-crown-6 compared with the $1: 1$ uronium nitrate/ 18 -crown-6 ratio in complex 4 . Secondly, only one hydrogen bond between the guanidinium ion and the crown was found (and a second one to the anion).

\subsection{CONFORMATIONAL FLEXIBILITY OF THE MACRORING}

Along with the different complexation schemes different conformations of the macro- 
ring are found. In the seven compounds studied four conformations are encountered: the 'biangular' $g^{+} g^{+} a a g^{-} a a^{-} a g^{-} g^{-} a a g^{+} a a g^{+} a$ conformation (complexes $\mathbf{1}$ and $\mathbf{2}$ and compound 7); the ' $C_{\mathrm{m}}$ ' conformation $\left(g^{+} g^{+} a a^{-} a a g^{+} a a^{-} g^{-} a g^{-} a a g^{+} a\right.$, highest occupied conformation of complex 3); the ' $C_{2}$ ' conformation $\left(g^{+} g^{+} a a^{-} a a^{-} g^{+} a g^{+} g^{+} a\right.$ $a g^{-} a a^{+} a$, lowest occupied conformation of complex 3, and complexes 4 and 5 ); and the ' $D_{3 \mathrm{~d}}$ ' conformation $\left(a g^{+} a{a g^{-}}^{-} a a^{+} a a g^{-} a a^{+} a a g^{-} a\right.$, complex 6). In the structures reported in the literature, mentioned above, the same conformations are found: the 'biangular' conformation (in the 1:4 thiourea complexes [6a, 6e], the $m$-chlorophenylurea complex [6d], one independent macroring in the $\mathrm{N}$-methylthiourea complex [3e], and in the guanidium nitrate complex [26]) and the $D_{3 \mathrm{~d}}$ conformation (in the $1: 2$ thiourea complex [6b], in the complex with $\mathrm{N}, \mathrm{N}^{\prime}$-dimethylthiourea $[6 \mathrm{c}]$, and in the second independent macroring in the N-methylthiourea complex [3e]).

All these conformations, except the $C_{2}$ one, can be viewed as 'ideal' crown ether conformations, i.e., superimposable on the diamond lattice, probably of low energy [31]. The simultaneous occurrence of the $C_{2}$ and the $C_{\mathrm{m}}$ conformations in complex 3 shows that the $C_{2}$ conformation, though not ideal, is closely related to an ideal conformation. Likewise, the fact that the $C_{2}$ conformation is adopted in complex 4, whereas the macroring in the closely related guanidinium nitrate complex with 18-crown-6 adopts the 'biangular' conformation [26], shows that the balance between these two conformations (and hence the complexation ratio of $1: 1$ or $1: 2$ in these complexes) must be very subtle. A molecular mechanics study by Uiterwijk et al. indeed showed that these three conformations have almost identical energies [32]. Moreover, the four conformations encountered in the seven compounds 1-7 are the lowest-energy conformations among hundreds of different conformations in polar environments (i.e., in the complexes) [32].

\section{Conclusions}

The complexation scheme of 18-membered crown ethers with urea and urea-derivatives depends on several factors, including: (1) the nature of the guests (neutral or charged); also, for charged guest complexes on (2) the way of introducing the charge (protonation or alkylation), and (3) the type of the anion involved.

Although the macroring in the different complexes may show some flexibility, the conformations encountered are closely related to one another and are clearly suited for this type of complexation.

\section{References}

1. (a) A. Barth and H.-J. Michel: Biochem. Physiol. Pflanz. 103 (1972).

(b) I. O. Sutherland: Chem. Soc. Rev. 15, 63 (1986).

2. (a) F. de Jong and D. N. Reinhoudt: Adv. Phys. Org. Chem. 17, 279 (1980).

(b) D. J. Cram and J. M. Cram: Acc. Chem. Res. 11, 8 (1978).

3. (a) A. van Zon, F. de Jong, D. N. Reinhoudt, G. J. Torny, and Y. Onwezen: Recl. Trav. Chim. Pays-Bas 100, 453 (1981).

(b) F. Vögtle, H. Sieger, and W. M. Müller: Top Curr. Chem. 98, 107 (1981).

(c) A. Elbasyouny, H. J. Brügge, K. von Deuten, M. Dickel, A. Knöchel, K. U. Koch, J. Kopf,

D. Melzer, and G. Rudolph: J. Am. Chem. Soc. 105, 6568 (1983).

(d) F. Vögtle and W. M. Müller: J. Incl. Phenom. 1, 369 (1983). 
(e) W. H. Watson, J. Galloy, D. A. Grossie, F. Vögtle, and W. M. Müller: J. Org. Chem. 49, 347 (1984).

(f) F. Vögtle, W. M. Müller, and W. H. Watson: Top. Curr. Chem. 125, 131 (1984).

(g) E. Weber, H.-P. Josel, H. Puff, and S. Franken: J. Org. Chem. 50, 3125 (1985).

4. C. J. Pedersen: J. Org. Chem. 36, 1690 (1971).

5. Incidentally in the literature adducts of urea and linear polyethers with a defined stoichiometry have been reported:

(a) G. Oepen and F. Vögtie: Jusius Liebigs Ann. Chem. 512 (1980).

(b) U. Heimann and F. Vögtle: Chem. Ber. 112, 3034 (1979).

(c) F. Vögtle, G. Oepen, and W. Rasshofer: Justus Liebigs Ann. Chem. 1577 (1979).

(d) K. K. Chacko, P. Narasimhan, and W. Saenger: Acta Crystallogr., Sect. C 40, 160 (1984).

6. See ref. [3] and references cited therein; complexes of crown ethers with urea-like neutral guests include those of 18 -crown-6 with thiourea in both $(1: 4)[6 \mathrm{a}]$ and $(1: 2)[6 \mathrm{~b}]$ ratios, with $\mathrm{N}$-methylthiourea $(1: 1)$ [3e], $N, N^{\prime}$ - dimethylthiourea $(1: 2)[6 \mathrm{c}]$ and $m$-chlorophenylurea $(1: 2)[6 \mathrm{~d}]$, and of diaza-18-crown-6 with thiourea $(1: 4)[6 \mathrm{e}]$ :

(a) G. Weber: J. Incl. Phenom. 1, 339 (1984).

(b) M. G. B. Drew, and D. G. Nicholson: Acta Crystallogr., Sect. C 41, 1358 (1985).

(c) G. Weber: ibid 39, 896 (1983).

(d) J. Weiler: Bull. Soc. Chim. Belg. 94, 1101 (1985).

(e) G. Weber: Acta Crystallogr., Sect. B 38, 2712 (1982).

7. (a) J. A. A. de Boer, D. N. Reinhoudt, S. Harkema, G. J. van Hummel, and F. de Jong: J. Am. Chem. Soc. 104, 4073 (1982).

(b) C. J. van Staveren, V. M. L. J. Aarts, P. D. J. Grootenhuis, J. van Eerden, S. Harkema, and D. N. Reinhoudt: J. Am. Chem. Soc. 108, 5271 (1986).

8. J. A. A. de Boer, J. W. H. M. Uiterwijk, J. Geevers, S. Harkema, and D. N. Reinhoudt: J. Org. Chem. 48, 4821 (1983).

9. J. W. H. M. Uiterwijk, C. J. van Staveren, D. N. Reinhoudt, H. J, den Hertog, Jr., L. Kruise, and S. Harkema: J. Org. Chem. 51, 1575 (1986).

10. Neutron diffraction: J. E. Worsham, and W. R. Busing: Acta Crystallogr., Sect. B 25, 572 (1969); X-ray diffraction: S. Harkema and D. Feil: ibid 25, 589 (1969).

11. V. M. L. J. Aarts, C. J. van Staveren, P. D. J. Grootenhuis, J. van Eerden, L. Kruise, S. Harkema, and D. N. Reinhoudt: J. Am. Chem. Soc. 108, 5035 (1986).

12. (a) V. M. L. J. Aarts, J. Geevers, D. N. Reinhoudt, W. Lengton, M. Bos, J. W. H. M. Uiterwijk, and S. Harkema: Tetrahedron 43,617 (1987); part of this work has been published in preliminary form in:

(b) J. W. H. M. Uiterwijk, S. Harkema, D. N. Reinhoudt, K. Daasvatn, H. J. den Hertog, Jr., and J. Geevers: Angew. Chem. Suppl. 1100 (1982); Angew. Chem., Int. Ed. Engl. 21, 450 (1982).

13. By analogy to the preparation of O-methyl-isouronium picrate as described by J. W. Janus: $J$. Chem. Soc. 3551 (1955).

14. S. Harkema: Ph.D. Thesis. Twente University of Technology, Enschede, The Netherlands, 1971.

15. R. Stewart and L. J. Mirenster: Can. J. Chem. 39, 401 (1961).

16. J. Dale and K. Daasvatn: J. Chem. Soc., Chem. Commun. 295 (1976).

17. H. Maeda, Y. Nakatsuji, and M. Okahara, J. Chem. Soc., Chem. Commun. 471 (1981).

18. G. Germain, P. Main, and M. M. Woolfson: Acta Crystallogr., Sect. A 27, 368 (1971).

19. W. R. Busing, K. O. Martin, and H. A. Levy: ORFLS, Report ORNL-TM-305. Oak Ridge National Laboratory: Oak Ridge, TN, 1962.

20. International Tables for X-ray Crystallography, Vol. IV, pp. 72-98. Kynoch Press: Birmingham, 1974 (Distr. D. Reidel, Dordrecht).

21. R. F. Stewart, E. R. Davidson, and W. T. Simpson: J. Chem. Phys. 42, 3175 (1965).

22. F. de Jong, D. N. Reinhoudt, and C. J. Smit: Tetrahedron Lett. 17, 1375 (1976).

23. D. Ph. Zollinger, M. Bos, A. M. W. van Veen-Blaauw, and W. E. van der Linden: Anal. Chim. Acta 161, 83 (1984).

24. D. N. Reinhoudt, J. A. A. de Boer, J. W. H. M. Uiterwijk, and S. Harkema: J. Org. Chem. 50, 4809 (1985).

25. J. W. H. M. Uiterwijk, S. Harkema, G. J. van Hummel, J. Geevers, and D. N. Reinhoudt: deta Crystallogr., Sect. B 38, 1862 (1982).

26. J. A. Bandy, M. R. Truter, J. N. Wingfield, and J. D. Lamb: J. Chem. Soc., Pcrkin Trans. 21025 (1981). 
27. A preliminary account of the structure of the $[: 5$ adduct of 18 -crown- 6 with urea (I) was given in: $S$. Harkema, G. J. van Hummel, K. Daasvatn, and D. N. Reinhoudt: $I$. Chem. Soc., Chem. Commun. 368 (1981); the structure of the $1: 1$ complex of 18 -crown-6 with uronium nitrate (4) was reported in preliminary form in ref. [12b].

28. C. K. Johnson: ORTEP, Report ORNL-3794. Oak Ridge National Laboratory: Oak Ridge, TN, 1965.

29. B. T. M. Willis and A. W. Pryor: Thermal Vibrations in Crystallography, pp. 101-102. Cambridge University Press: Cambridge, 1975.

30. M. Herceg and R. Weiss: Bull. Soc. Chim. Fr. 549 (1972).

31. J. W. H. M. Uiterwijk, S. Harkema, B.W. van der Waal, F. Göbel, and H. T. M. Nibbeling: J. Chem. Soc. Perkin Trans. 21843 (1983).

32. (a) J. W. H. M. Uiterwijk, S. Harkema, and D. Feil: J. Chem. Soc, Perkin Trans. 2721 (1987).

(b) J. W. H. M. Uiterwijk: Ph.D. Thesis. Twente University of Technology, Enschede, The Netherlands, 1985. 